\title{
Type I photosynthetic reaction center in an anoxygenic phototrophic member of the Chloroflexota
}

Tsuji $\mathrm{JM}^{1 \dagger *}$, Shaw NA ${ }^{1}$, Nagashima $\mathrm{S}^{2 \ddagger}$, Venkiteswaran $\mathrm{JJ}^{1,3}$, Schiff $\mathrm{SL}^{1}$, Hanada $\mathrm{S}^{2 \S}$, Tank $\mathrm{M}^{2,4}$, Neufeld $\mathrm{JD}^{1 *}$

$5 \quad{ }^{1}$ University of Waterloo, 200 University Avenue West, Waterloo, Ontario, Canada, N2L 3G1

${ }^{2}$ Tokyo Metropolitan University, 1-1 Minami-osawa, Hachioji, Tokyo, Japan, 192-0397

${ }^{3}$ Wilfrid Laurier University, 75 University Avenue West, Waterloo, Ontario, Canada, N2L 3C5

${ }^{4}$ Leibniz Institute DSMZ-German Collection of Microorganisms and Cell Cultures GmbH, Inhoffenstrasse 7B, 38124 Braunschweig, Germany

10

${ }^{\dagger}$ Current address: Institute of Low Temperature Science, Hokkaido University, Kita-19, Nishi-8, Kita-ku, Sapporo, Japan, 060-0819

${ }^{\ddagger}$ Current address: Kanagawa University, 2946 Tsuchiya, Hiratsuka, Kanagawa, Japan, 259-1293

${ }^{\S}$ Current address: Bioproduction Research Institute, National Institute of Advanced Industrial Science and

15 Technology (AIST), Tsukuba Central 6, 1-1-1 Higashi, Tsukuba, Ibaraki, Japan, 305-8566

*Corresponding authors: jackson.tsuji@uwaterloo.ca; jneufeld@uwaterloo.ca

Keywords: Chloroflexota; Chloroflexi; filamentous anoxygenic phototroph; boreal lakes; anoxygenic photoautotrophy; evolution of photosynthesis; enrichment cultivation; metatranscriptomics 


\section{Summary}

Phototrophic members of the Chloroflexota phylum are enigmas in the evolution of phototrophy. Although all previously characterized phototrophic Chloroflexota members use a Type II reaction center (RCII) to perform light energy conversion ${ }^{1,2}$, many members host chlorosomes, a light-harvesting apparatus only otherwise reported among Type I reaction center (RCI)-containing phototrophs ${ }^{3}$. Here we report the discovery and

25 cultivation of "Candidatus Chlorohelix allophototropha", the first known member of the Chloroflexota to use $\mathrm{RCI}$, rather than RCII, for phototrophy. Cultivated from an iron-rich Boreal Shield lake, "Ca. Chx. allophototropha" cells contain chlorosomes and encode a novel fifth clade of RCI protein. Phylogenomic data demonstrate that " $\mathrm{Ca}$. Chx. allophototropha" shares closest ancestry to RCII-utilizing relatives for multiple photosynthesis accessory genes, including chlorosomes. Thus, we propose that the enigmatic distribution of chlorosomes in the Chloroflexota phylum resulted from genetic interaction of RCI- and RCII-utilizing Chloroflexota members. Although undetected previously, metatranscriptome data demonstrate that RCI-utilizing Chloroflexota members can be among the most active populations in illuminated anoxic waters of Boreal Shield lakes, which number in the millions globally ${ }^{4}$. Our results establish the Chloroflexota as the only bacterial phylum containing RCI- and RCII-utilizing anoxygenic phototrophs, providing new context for understanding

35 the origins of phototrophic life on Earth.

\section{Main}

Chlorophyll-based phototrophy drives the majority of global carbon fixation ${ }^{5}$ and relies on a Type I (RCI) and/or Type II (RCII) photosynthetic reaction center for light energy conversion ${ }^{2}$. Although oxygenic

40 phototrophy is the dominant chlorophototrophic process today ${ }^{6}$, most phototrophic genetic diversity is distributed among anoxygenic phototrophs affiliated with seven bacterial phyla ${ }^{1}$. Unlike oxygenic phototrophs, which use RCII and RCI in tandem as Photosystem II (PSII) and Photosystem I (PSI), respectively, anoxygenic phototrophs consistently use either RCI or RCII for phototrophy ${ }^{2}$. The photosynthetic reaction centers of both oxygenic and anoxygenic phototrophs have been extensively characterized to understand their physiological and evolutionary properties (reviewed in ${ }^{2,7,8}$ ). Although it appears that RCI and RCII diverged early in the evolution of life $^{9}$, it remains unclear how they became incorporated in a single organism, in the case of oxygenic phototrophs, yet appear to be well separated otherwise within the tree of life ${ }^{10,11}$. Outside of members of the Cyanobacteria phylum (and, by extension, chloroplasts in eukaryotes), RCI and RCII-utilizing phototrophs appear distantly related, belonging to separate bacterial phyla and generally using divergent photosynthesis accessory genes ${ }^{1}$. Although some have speculated that genetic interactions of RCI and RCII ought to have occurred among anoxygenic phototrophs over evolutionary time ${ }^{11,12}$, no direct published evidence has existed to support this hypothesis.

We sampled the anoxic water column of Lake 227, an iron-rich Boreal Shield lake ${ }^{4}$, and cultivated the first known member of the Chloroflexota (formerly Chloroflexi) phylum to use RCI for phototrophy (Fig. 1;

55 Supplementary Note 1). Given that only RCII-utilizing Chloroflexota members were known previously ${ }^{1,13,14}$, this 
bioRxiv preprint doi: https://doi.org/10.1101/2020.07.07.190934; this version posted August 7, 2021. The copyright holder for this preprint (which was not certified by peer review) is the author/funder, who has granted bioRxiv a license to display the preprint in perpetuity. It is made available under aCC-BY-NC-ND 4.0 International license.



Fig. 1 | Type I reaction center-based phototrophy in " $\mathrm{Ca}$. Chlorohelix allophototropha". a, Overview of the eight bacterial phyla containing known chlorophototrophs. A class-level summary of the Genome Taxonomy Database (GTDB) bacterial reference tree (release 89) is shown, with chlorophototrophic phyla highlighted based on the photosynthetic reaction center utilized. b. Maximum likelihood phylogeny of Type I reaction center primary sequences. Ultrafast bootstrap values for major branch points are shown out of 100 based on 1000 replicates, and the scale bar shows the expected proportion of amino acid change across the 548-residue masked sequence alignment (Extended Data Table 2). Chlorophototrophic lineages are summarized by order name. The placement of the PscA-like sequence of " $\mathrm{Ca}$. Chx. allophototropha" is indicated by a black dot. All clades above they grey dot utilize chlorosomes. c, Predicted tertiary structure of the novel " $\mathrm{Ca}$. Chlorohelix allophototropha" PscA-like primary sequence based on homology modelling. The six N-terminal and five C-terminal transmembrane helices expected for RCI are coloured in red and tan, respectively. $\mathbf{d}$, Epifluorescence microscopy image of " $\mathrm{Ca}$. Chx. allophototropha" cells. Cell autofluorescence is shown with light excitation and detection wavelengths optimized for bacteriochlorophyll $c$. e, Transmission electron microscopy image showing a

70 longitudinal section of " $\mathrm{Ca}$. Chx. allophototropha" cells. An example chlorosome is marked with an arrow. f, Absorption spectrum (350-850 nm) of an acetone:methanol extract of pigments from the " $\mathrm{Ca}$. Chx. allophototropha" culture. Major absorption peaks are marked by dashed lines. Scale bars in panels $\mathbf{d}$ and e represent $10 \mu \mathrm{m}$ and $0.1 \mu \mathrm{m}$, respectively. 
novel phototroph breaks established paradigms that each bacterial phylum of anoxygenic phototrophs is associated with a single type of photosynthetic reaction center (Fig. 1a) ${ }^{1,2,7}$. We did not detect the pufLM genes associated with RCII in the highly complete genome of the organism or in the enrichment culture metagenome from which that genome was derived (Supplementary Note 2; Extended Data Fig. 1). Instead, we detected a remote homolog of the $p s c A$ gene, encoding the RCI protein, on a $3.29 \mathrm{Mb}$ scaffold that was flanked by other genes classified to the Chloroflexota phylum (Supplementary Data 1). Based on a maximum-likelihood amino acid sequence phylogeny (Fig. 1b; Extended Data Fig. 2), the PscA-like predicted protein of the novel

80 phototroph represents a distinct fifth clade of RCI/PSI protein and thus was not acquired by recent lateral gene transfer from known phototrophic clades ${ }^{15}$. Homology modelling indicated that the novel phototroph's PscA-like protein contained the expected six N-terminal transmembrane helices for coordinating antenna pigments and five C-terminal transmembrane helices involved in core electron $\operatorname{transfer}^{16}$ (Fig. 1c), supporting its functional role. We did not detect a cyanobacterial psaB-like paralog of the RCI gene, implying that the novel phototroph uses a homodimeric RCI complex like other known anoxygenic phototrophs ${ }^{2}$.

In line with a phototrophic growth mode, cells of the novel strain grew as pigmented filaments (Fig. 1d) and contained light-harvesting chlorosomes (Fig. 1e; Extended Data Fig. 3), with the dominant pigment being bacteriochlorophyll $c$ based on absorption spectrum data (Fig. 1f; see Supplementary Note 3$)^{17}$. We also enriched a second phototrophic Chloroflexota member whose genome encoded a pscA-like gene belonging to the same

90 fifth RCI/PSI clade (Fig. 1b), supporting that the novel RCI gene is associated with Chloroflexota members (Supplementary Notes 1-2). Combined with metatranscriptome data (see below), our findings provide robust evidence that the novel Chloroflexota member relies on the detected RCI to perform phototrophy. Thus, we provisionally classify the novel phototroph as, "Candidatus Chlorohelix allophototropha" strain L227-S17 (Chlo.ro.he'lix. Gr. adj. chloros, green; Gr. fem. n. helix, spiral; N.L. fem. n. Chlorohelix, a green spiral.

95 al.lo.pho.to.tro'pha. Gr. adj. allos, other, different; Gr. n. phos, -otos, light; N.L. suff. -tropha, feeding; N.L. fem. adj. allophototropha, phototrophic in a different way), the first cultivated member of the provisional order “Candidatus Chloroheliales" within the Chloroflexia class (Supplementary Note 4).

Phylogenomic analyses of the " $\mathrm{Ca}$. Chx. allophototropha" genome revealed several phototrophyassociated genes that were highly divergent from those of known phototrophs (Fig. 2; Extended Data Table 1;

100 Supplementary Data 2). We detected a homolog of fmoA, encoding the Fenna-Matthews-Olson (FMO) protein involved in energy transfer from chlorosomes to $\mathrm{RCI}^{18}$, with $21-26 \%$ predicted amino acid identity to known FmoA sequences (Extended Data Fig. 4). This finding makes the Chloroflexota the third known phylum to use the FMO protein in phototrophy ${ }^{19}$. Intriguingly, the FmoA predicted primary sequence lacked the Cys49 and Cys353 residues thought to be crucial for quenching energy transfer ${ }^{20}$. Matching physiological observations, we 105 detected a homolog of the key chlorosome structural gene $\operatorname{cs} m A^{12,21}$ that had $\sim 33 \%$ identity at the predicted amino acid level to the CsmA primary sequence of Chloroflexus aurantiacus J-10-fl (Fig. 2), along with homologs of $\operatorname{csm} M$ and $\operatorname{csm} Y^{12}$. The potential CsmA homolog notably lacked the His 25 thought to be required for bacteriochlorophyll $a$ binding ${ }^{22}$ (Extended Data Fig. 5). In place of Alternative Complex III used by 
bioRxiv preprint doi: https://doi.org/10.1101/2020.07.07.190934; this version posted August 7, 2021. The copyright holder for this preprint (which was not certified by peer review) is the author/funder, who has granted bioRxiv a license to display the preprint in perpetuity. It is made available under aCC-BY-NC-ND 4.0 International license.



Fig. 2 Genomic potential for phototrophy among members of the Chloroflexota phylum. A maximum likelihood phylogeny of key members of the phylum is shown on the left based on a set of 74 concatenated core bacterial proteins. Ultrafast bootstrap values are shown out of 100 based on 1000 replicates, and the scale bar shows the expected proportion of amino acid change across the 11 613-residue masked sequence alignment (Extended Data Table 2). The " $\mathrm{Ca}$.

115 Chloroheliales" clade is highlighted in orange, and genomes recovered in this study are in bold. On the right, a heatmap shows the presence/absence of genes involved in photosynthesis or related processes based on bidirectional BLASTP. The intensity of the fill colour of each tile corresponds to the percent identity of the BLASTP hit. Query sequences were derived from the genomes of "Ca. Chlorohelix allophototropha", Chloroflexus aurantiacus, Roseiflexus castenholzii, or Oscillochloris trichoides, and the query organism used for each gene is marked by bolded outlines on the heatmap.

120 GenBank accession numbers of sequences associated with the novel " $\mathrm{Ca}$. Chloroheliales" members enriched in this work are summarized in Extended Data Table 1, and raw results of bidirectional BLASTP are summarized in Supplementary Data 2. Orders containing potential phototrophs are labelled on the right of the heatmap. All genomes in the phylogeny encoded at least $50 \%$ of the core 74 protein set except for Chloroflexi RRmetagenome_bin16 and "Ca. Thermofonsia bin CP2-42A", which encoded 25 and 34 of the proteins, respectively. 
Chloroflexales members for photosynthetic electron transport ${ }^{23}$, we found a cytochrome $b_{6} f$-like gene cluster including a putative di-heme cytochrome $c$ as described for Heliobacterium modesticaldum ${ }^{24}$ (Supplementary Note 2). Moreover, we detected genes for the Calvin-Benson-Bassham (CBB) cycle for carbon fixation in the “Ca. Chx. allophototropha" genome, including a deep-branching Class IC/ID "red type" $c b b L$ gene $^{25}$

130 representing the large subunit of RuBisCO (Extended Data Fig. 6) and the $c b b P$ gene encoding phosphoribulokinase $^{26}$ (Fig. 2). We did not detect genomic potential for the 3-hydroxypropionate bicycle, which is used for carbon fixation by some RCII-utilizing Chloroflexales members ${ }^{27}$. "Ca. Chx. allophototropha" thus represents the first known RCI-utilizing phototroph with potential for carbon fixation via the CBB cycle, with metatranscriptome data supporting that $\mathrm{RuBisCO}$ is actively used for photosynthesis.

135 Although previously overlooked, environmental metagenome and metatranscriptome data demonstrate that relatives of " $\mathrm{Ca}$. Chx. allophototropha" are important phototrophic community members in iron-rich Boreal Shield lakes (Fig. 3). Surveying the water columns of eight seasonally anoxic Boreal Shield lakes at the International Institute for Sustainable Development Experimental Lakes Area (IISD-ELA) using metagenome sequencing, we recovered 756 dereplicated metagenome-assembled genomes (MAGs) having an average 140 completeness and contamination of $82.7 \%$ and $2.1 \%$, respectively. From these, we identified two RCI-encoding MAGs affiliated with the "Ca. Chloroheliales" order (Supplementary Note 5). Short-read mapping of metagenome sequences to these MAGs demonstrated their widespread distribution within the illuminated anoxic zones of four lakes (i.e., Lakes 221, 222, 227, and 304), with relative abundances of up to 1.32\% (Supplementary Data 3), although the relative abundance and activity of these MAGs were low in Lake 227 (Supplementary Note

145 5). Importantly, metatranscriptome data available for the anoxic water columns of Lakes 221 and 304 showed that the "Ca. Chx. allophototropha" relatives were highly photosynthetically active. In Lake 221, the two MAGs recruited a cumulative $3.3 \%$ of mapped RNA reads, and in Lake 304, "Ca. Chloroheliales" bin ELA729 was among the top ten highest RNA read-recruiting MAGs in the dataset (Fig. 3a). Both MAGs were found to have high expression of the $p s c A$-like RCI gene, the $f m o A$ gene, and the $c b b L S$ genes encoding RuBisCO, with " $C a$.

150 Chlorohelix" bin ELA319 (Lake 221) having all of these phototrophy-associated genes within the top 30 most highly expressed genes in its genome (Fig. 3b; Extended Data Fig. 7). Moreover, the two MAGs co-occurred with highly active RCII-encoding MAGs associated with the Chloroflexales order (Fig. 3a), which suggests that RCI- and RCII-utilizing Chloroflexota members can occupy the same or closely related niche space. Given that Boreal Shield lakes number in the tens of millions globally, and that an estimated $15 \%$ develop seasonally anoxic 155 conditions $^{4}$, relatives of " $\mathrm{Ca}$. Chx. allophototropha" could be responsible for substantial and previously unreported contributions to the freshwater biogeochemistry.

Based on phylogenomic data, the proposed " $\mathrm{Ca}$. Chloroheliales" order containing " $\mathrm{Ca}$. Chx. allophototropha" places directly sister to the Chloroflexales order containing the canonical RCII-utilizing phototrophic clade in the phylum (Fig. 2). The phylogenetic proximity of these RCI- and RCII-utilizing groups is 160 unprecedented and contrasts with the observations of current literature ${ }^{1,2,7}$. Overlapping environmental niches between RCII-utilizing Chloroflexota members, which are known to inhabit mesophilic aquatic 
a

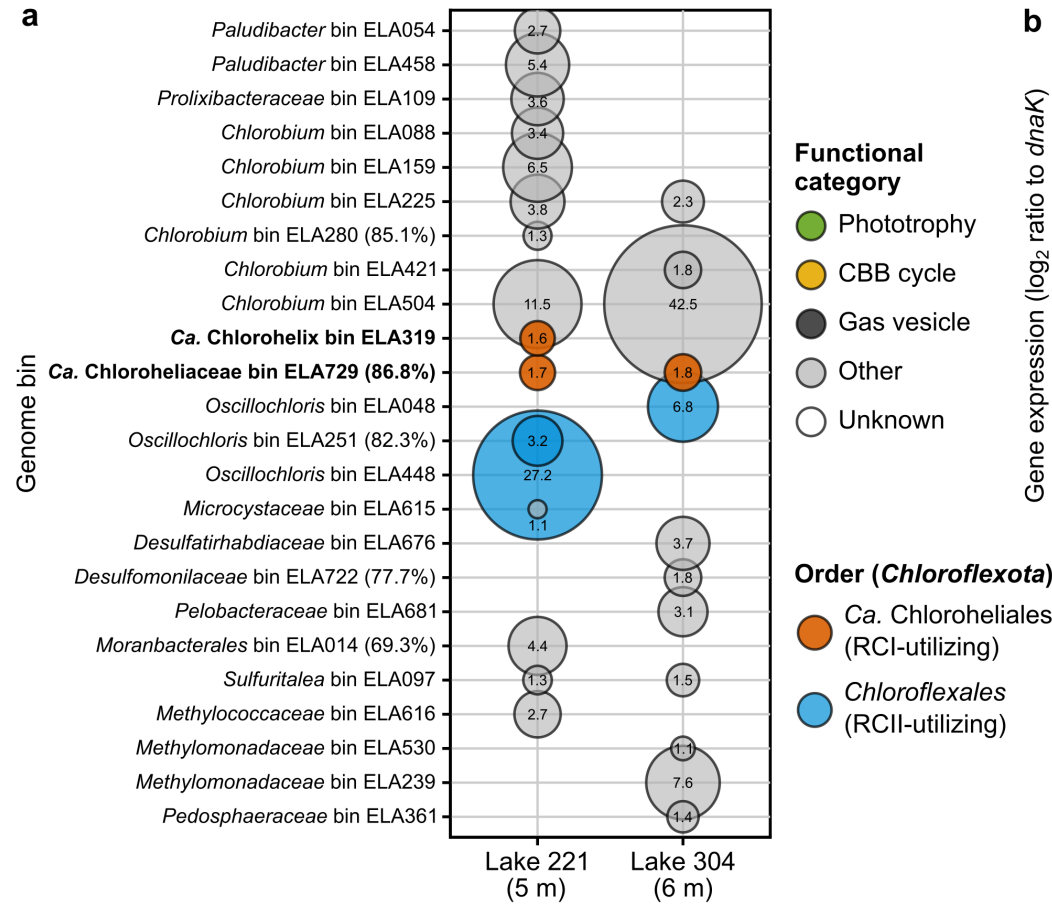

b

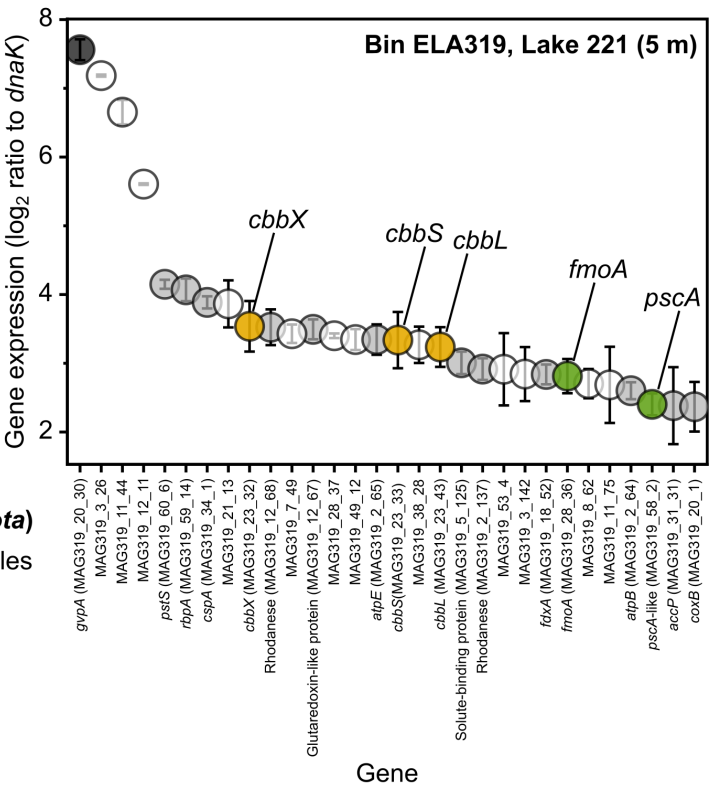

Fig. 3 | Environmental activity of " $\mathrm{Ca}$. Chx. allophototropha" relatives in Boreal Shield lakes. a, Bubble plot showing active microbial populations in the water columns of two Boreal Shield lakes at the IISD-ELA based on read mapping of metatranscriptome data (from July 2018) to metagenome-assembled genomes (MAGs). The size of each bubble represents the mean "relative expression" $(\mathrm{n}=3)$ of each MAG, as described in the methods, and MAGs having $>1 \%$ relative expression are shown. (See Supplementary Data 4 for standard deviations, which were on average $7.0 \%$ of the mean.) All MAGs shown had $<5 \%$ estimated contamination, and for any MAGs with $<90 \%$ estimated completion, the completion metric is shown beside the MAG ID. b, Normalized gene expression of " $\mathrm{Ca}$. Chlorohelix bin ELA319" based on Lake 221 metatranscriptome data. The top 30 protein-coding genes with highest normalized expression values are shown. Error bars represent the standard deviation of the $\log _{2}$ expression ratio based on triplicate metatranscriptomes $(n=3)$. Genes potentially involved in phototrophy, carbon fixation, and buoyancy are highlighted. Highly expressed genes based on Lake 304 metatranscriptome data and read mapping to bin ELA729 are shown in Extended Data Fig. 7, and normalized expression values for all genes, along with their sequences and annotations, are included in Supplementary Data 5.

environments $^{28,29}$, and RCI-utilizing “ $\mathrm{Ca}$. Chx. allophototropha” relatives could readily facilitate the genetic interaction of these chlorophototroph clades. We probed the phylogenetic history of several photosynthesisassociated genes of " $\mathrm{Ca}$. Chx. allophototropha", in the context of known phototrophs, to explore whether such genetic interaction might have taken place (Fig. 4). In a maximum likelihood phylogeny of the chlorosome structural protein CsmA, “Ca. Chloroheliales” and RCII-utilizing Chloroflexota sequences grouped directly sister to one another (Fig. 4a; Extended Data Fig. 8). Similarly, using maximum likelihood phylogenies of the (bacterio)chlorophyll synthesis proteins BchIDH/ChlIDH (Fig. 4b; Extended Data Fig. 9) and BchLNB/ChlLNB/BchXYZ (Extended Data Fig. 10), we observed that " $\mathrm{Ca}$. Chloroheliales" sequences placed immediately adjacent to the sister grouping of RCII-utilizing Chloroflexota and RCI-utilizing Chlorobia members. Although the long branch lengths in these phylogenies complicate inferring ancestral relationships, the consistent close placement of RCI- and RCII-utilizing Chloroflexota clades in these phylogenies provides first evidence that the two clades have a shared genetic history of phototrophy despite using different core reaction centers. Indeed, the presence of chlorosomes within phototrophic Chloroflexota members has been enigmatic 

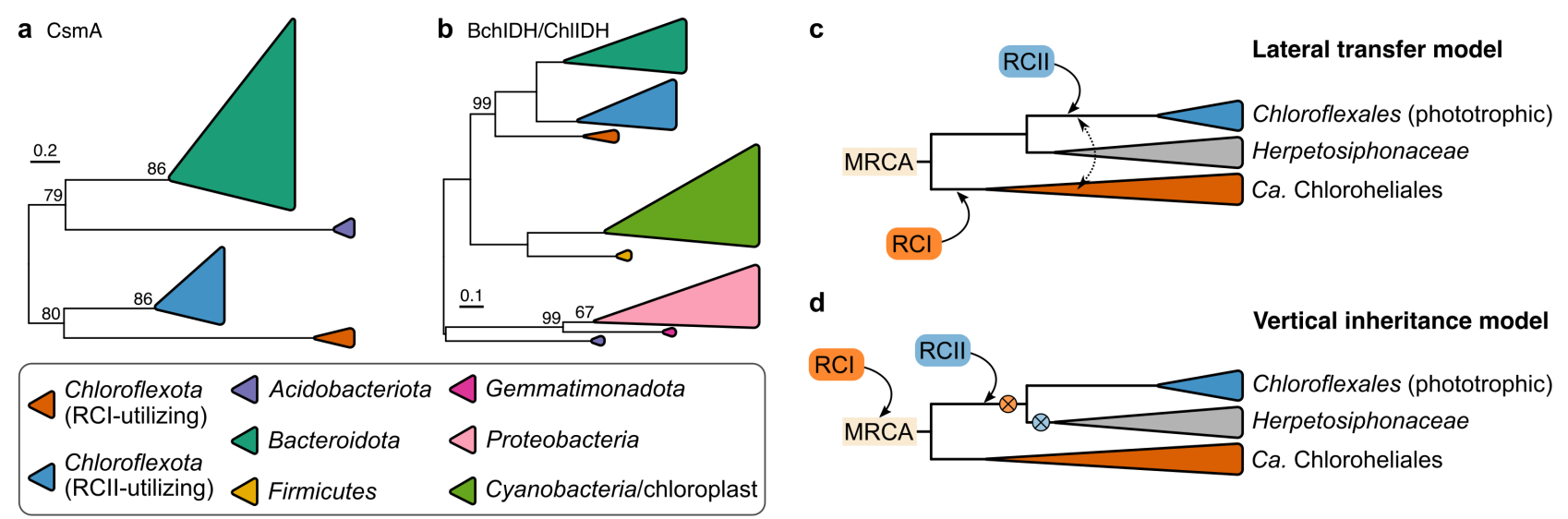

Fig. 4 | Evolution of phototrophy within the Chloroflexota phylum. a-b, Maximum likelihood phylogenies of the chlorosome structural protein CsmA (a) and (bacterio)chlorophyll synthesis proteins BchIDH/ChlIDH (b). The phylogenies are midpoint rooted, and ultrafast bootstrap values less than 100/100 are shown. Scale bars represent the expected proportion of amino acid change across the masked sequence alignments (see Extended Data Table 2). Detailed versions of the same phylogenies are shown in Extended Data Figs. 8-9. c-d, Proposed models of phototrophy evolution within the phylum. In the lateral transfer model (c), ancestors of the " $\mathrm{Ca}$. Chloroheliales" and Chloroflexales orders independently obtained RCI and RCII, respectively. Subsequent lateral gene transfer between members of the two orders allowed for members of both orders to encode related photosynthesis accessory genes (e.g., for chlorosome structure). In the vertical inheritance model (d), the most recent common ancestor (MRCA) of the "Ca. Chloroheliales" and Chloroflexales orders encoded either RCI or RCII and several photosynthesis accessory genes (e.g., for chlorosome structure). Genes for the alternate reaction center were subsequently acquired by descendants of that ancestor, followed by loss of the original reaction center. Phototrophy genes were then lost in ancestors of certain clades, such as the non-phototrophic

205 Herpetosiphonaceae family within the Chloroflexales order. The schematic depicts the MRCA having RCI, followed by acquisition of RCII, but alternative orders of photosynthesis gene gain/loss, or multiple gene gain/loss events, are also possible in the vertical inheritance model.

210 because these light-harvesting organelles are only otherwise found in RCI-utilizing phototrophs ${ }^{3,12,21}$. The discovery of "Ca. Chx. allophototropha” resolves this enigma if chlorosomes in RCII-utilizing Chloroflexota members were sourced from ancestors of the RCI-utilizing "Ca. Chx. allophototropha”, or vice versa.

Our phylogenomic data leave open the possibility of two broad modes of genetic interaction between RCIand RCII-utilizing Chloroflexota members. First, these two phototrophic Chloroflexota groups could have

215 obtained their reaction centers independently and subsequently exchanged photosynthesis accessory genes, such as chlorosome-associated genes, via lateral gene transfer (Fig. 4c). Lateral gene transfer has been previously observed to play a role in the evolution of RCII-driven phototrophy in the phylum ${ }^{30}$. Given that chlorosomes are associated only with RCI-utilizing phototrophs outside the Chloroflexota, it would follow that chlorosomes were laterally transferred from RCI- to RCII-utilizing Chloroflexota members. Alternatively, RCI- and RCII-utilizing

220 Chloroflexota members could share a common phototrophic ancestor and could have inherited chlorosomes and bacteriochlorophyll synthesis genes vertically from that ancestor (Fig. 4d). Consistent basal placement of “ $\mathrm{Ca}$. Chloroheliales"-associated photosynthesis accessory gene sequences compared to those of RCII-utilizing Chloroflexota members implies that the common ancestor contained RCI (Fig. 4a-b), although the presence of a pscA-fmoA gene cluster in the genome of one of our enriched " $\mathrm{Ca}$. Chloroheliales" members could also imply more recent acquisition of RCI in this clade (Supplementary Note 2). The vertical inheritance scenario leaves open the intriguing possibility that a common ancestor of RCI- and RCII-utilizing Chloroflexota members used 
RCI and RCII simultaneously, in a manner analogous to Cyanobacteria members, followed by a "genetic displacement" event of the other reaction center, as has been speculated previously ${ }^{12}$. Either the lateral gene transfer or vertical inheritance scenario potentially clarifies the origin of chlorosomes among RCII-utilizing

Chloroflexota members and demonstrates the possibility for close genetic interaction of RCI and RCII within the Chloroflexota.

By cultivating the first-known RCI-utilizing phototroph within the Chloroflexota phylum, we demonstrate that RCI and RCII can be used by closely related anoxygenic phototrophs, in contrast to established paradigms. Discovering “ $\mathrm{Ca}$. Chx. allophototropha” demonstrates the possibility for massive photosynthesis gene

movements over evolutionary time, which has been required by many evolutionary models previously but has been sparsely demonstrated in nature ${ }^{8,15}$. Cultivation of " $\mathrm{Ca}$. Chx. allophototropha" substantially expands the known diversity of chlorophototrophy, with implications for the biochemistry, evolution, and ecology of photosynthesis.

\section{References}

1. Thiel, V., Tank, M. \& Bryant, D. A. Diversity of chlorophototrophic bacteria revealed in the omics era. Annu Rev Plant Biol 69, 21-49 (2018).

2. Fischer, W. W., Hemp, J. \& Johnson, J. E. Evolution of oxygenic photosynthesis. Annu Rev Earth Pl Sc 44, 647-683 (2016).

245 3. Hohmann-Marriott, M. F. \& Blankenship, R. E. Hypothesis on chlorosome biogenesis in green photosynthetic bacteria. FEBS Lett 581, 800-803 (2007).

4. Schiff, S. L. et al. Millions of Boreal Shield lakes can be used to probe Archaean Ocean biogeochemistry. Sci Rep 7, 46708 (2017).

5. Field, C. B., Behrenfeld, M. J., Randerson, J. T. \& Falkowski, P. Primary production of the biosphere:

$250 \quad$ integrating terrestrial and oceanic components. Science 281, 237-240 (1998).

6. Raven, J. A. Contributions of anoxygenic and oxygenic phototrophy and chemolithotrophy to carbon and oxygen fluxes in aquatic environments. Aquat Microb Ecol 56, 177-192 (2009).

7. Hohmann-Marriott, M. F. \& Blankenship, R. E. Evolution of photosynthesis. Annu Rev Plant Biol 62, 515-548 (2011).

255 8. Martin, W. F., Bryant, D. A. \& Beatty, J. T. A physiological perspective on the origin and evolution of photosynthesis. FEMS Microbiol Rev 42, 205-231 (2018).

9. Sadekar, S., Raymond, J. \& Blankenship, R. E. Conservation of distantly related membrane proteins: photosynthetic reaction centers share a common structural core. Mol Biol Evol 23, 2001-2007 (2006).

10. Olson, J. M. \& Blankenship, R. E. Thinking about the evolution of photosynthesis. in Discoveries in Photosynthesis (eds. Govindjee, Beatty, J. T., Gest, H. \& Allen, J. F.) 1073-1086 (Springer Netherlands, 2005). doi:10.1007/1-4020-3324-9_95.

11. Cardona, T. Thinking twice about the evolution of photosynthesis. Open Biol 9, 180246 (2019).

12. Bryant, D. A. et al. Comparative and functional genomics of anoxygenic green bacteria from the taxa Chlorobi, Chloroflexi, and Acidobacteria. in Functional Genomics and Evolution of Photosynthetic Systems (eds. Burnap, R. \& Vermaas, W.) 47-102 (Springer Netherlands, 2012). doi:10.1007/978-94-0071533-2_3.

13. Pierson, B. K. \& Castenholz, R. W. A phototrophic gliding filamentous bacterium of hot springs, Chloroflexus aurantiacus, gen. and sp. nov. Arch Microbiol 100, 5-24 (1974). 
14. Hanada, S. The phylum Chloroflexi, the family Chloroflexaceae, and the related phototrophic families Oscillochloridaceae and Roseiflexaceae. in The Prokaryotes: Other Major Lineages of Bacteria and The Archaea (eds. Rosenberg, E., DeLong, E. F., Lory, S., Stackebrandt, E. \& Thompson, F.) 515-532 (Springer Berlin Heidelberg, 2014). doi:10.1007/978-3-642-38954-2_165.

15. Zeng, Y., Feng, F., Medová, H., Dean, J. \& Kobližzek, M. Functional type 2 photosynthetic reaction centers found in the rare bacterial phylum Gemmatimonadetes. Proc Natl Acad Sci USA 111, 7795-7800 (2014).

16. Gisriel, C. et al. Structure of a symmetric photosynthetic reaction center-photosystem. Science 357, 10211025 (2017).

17. Overmann, J. \& Garcia-Pichel, F. The phototrophic way of life. in The Prokaryotes: Prokaryotic Communities and Ecophysiology (eds. Rosenberg, E., DeLong, E. F., Lory, S., Stackebrandt, E. \& Thompson, F.) 203-257 (Springer Berlin Heidelberg, 2013). doi:10.1007/978-3-642-30123-0_51.

280 18. Olson, J. M. The FMO protein. in Discoveries in Photosynthesis (eds. Govindjee, Beatty, J. T., Gest, H. \& Allen, J. F.) 421-427 (Springer Netherlands, 2005). doi:10.1007/1-4020-3324-9_40.

19. Bryant, D. A. et al. Candidatus Chloracidobacterium thermophilum: an aerobic phototrophic Acidobacterium. Science 317, 523-526 (2007).

20. Orf, G. S. et al. Evidence for a cysteine-mediated mechanism of excitation energy regulation in a photosynthetic antenna complex. Proc Natl Acad Sci USA 113, E4486-E4493 (2016).

21. Frigaard, N.-U. \& Bryant, D. A. Seeing green bacteria in a new light: genomics-enabled studies of the photosynthetic apparatus in green sulfur bacteria and filamentous anoxygenic phototrophic bacteria. Arch Microbiol 182, 265-276 (2004).

22. Pedersen, M. Ø., Linnanto, J., Frigaard, N.-U., Nielsen, N. Chr. \& Miller, M. A model of the proteinpigment baseplate complex in chlorosomes of photosynthetic green bacteria. Photosynth Res 104, 233243 (2010).

23. Tang, K.-H. et al. Complete genome sequence of the filamentous anoxygenic phototrophic bacterium Chloroflexus aurantiacus. BMC Genomics 12, 334 (2011).

24. Ducluzeau, A. L., Chenu, E., Capowiez, L. \& Baymann, F. The Rieske/cytochrome $b$ complex of Heliobacteria. Biochim Biophys Acta, Bioenerg 1777, 1140-1146 (2008).

25. Tourova, T. P. et al. Phylogeny of anoxygenic filamentous phototrophic bacteria of the family Oscillochloridaceae as inferred from comparative analyses of the rrs, cbbL, and nifH genes. Microbiology 75, 192-200 (2006).

26. Tabita, F. R. The biochemistry and molecular regulation of carbon dioxide metabolism in cyanobacteria. in The Molecular Biology of Cyanobacteria (ed. Bryant, D. A.) 437-467 (Springer, 1994). doi:10.1007/97894-011-0227-8_14.

27. Shih, P. M., Ward, L. M. \& Fischer, W. W. Evolution of the 3-hydroxypropionate bicycle and recent transfer of anoxygenic photosynthesis into the Chloroflexi. Proc Natl Acad Sci USA 114, 10749-10754 (2017).

28. Gorlenko, V. M. et al. Candidatus 'Chloroploca asiatica' gen. nov., sp. nov., a new mesophilic filamentous anoxygenic phototrophic bacterium. Microbiology 83, 838-848 (2014).

29. Gaisin, V. A. et al. 'Candidatus Viridilinea mediisalina', a novel phototrophic Chloroflexi bacterium from a Siberian soda lake. FEMS Microbiol Lett 366, fnz043 (2019).

30. Ward, L. M., Hemp, J., Shih, P. M., McGlynn, S. E. \& Fischer, W. W. Evolution of phototrophy in the Chloroflexi phylum driven by horizontal gene transfer. Front Microbiol 9, 260 (2018). 


\section{Methods}

\section{Enrichment cultivation of "Ca. Chx. allophototropha"}

To culture novel anoxygenic phototrophs, we sampled Lake $227\left(49.69^{\circ} \mathrm{N}, 93.69^{\circ} \mathrm{W}\right)$, a seasonally anoxic and ferruginous Boreal Shield lake at the International Institute for Sustainable Development

315 Experimental Lakes Area (IISD-ELA). The IISD-ELA sampling site (49.50-49.75 $\left.\mathrm{N}, 93.50-94.00^{\circ} \mathrm{W}\right)$, located near Kenora, Canada, has been described in detail previously ${ }^{4,31-34}$. Lake 227 develops up to $\sim 100 \mu \mathrm{M}$ concentrations of dissolved ferrous iron in its anoxic water column ${ }^{4}$, and anoxia is more pronounced than expected naturally due to the long-term experimental eutrophication of the lake $\mathrm{e}^{35}$. We collected water from the illuminated portion of the upper anoxic zone of Lake 227 in September 2017, at 3.88 and $5.00 \mathrm{~m}$ depth, and

320 transported this water to the laboratory under anoxic and chilled conditions in $120 \mathrm{~mL}$ glass serum bottles sealed with black rubber stoppers (Geo-Microbial Technology Company; Ochelata, Oklahoma, USA).

Water was supplemented with $2 \% \mathrm{v} / \mathrm{v}$ of a freshwater medium containing $8 \mathrm{mM}$ ferrous chloride ${ }^{36}$ and was distributed under anoxic conditions into $120 \mathrm{~mL}$ glass serum bottles, also sealed with black rubber stoppers (Geo-Microbial Technology Company), that had a headspace of dinitrogen gas at $1.5 \mathrm{~atm}$ final pressure. Bottles 325 were spiked with a final concentration of $50 \mu \mathrm{M}$ Diuron or 3-(3,4-dichlorophenyl)-1,1-dimethylurea (DCMU); Sigma-Aldrich; St. Louis, Missouri, USA) to block oxygenic phototrophic activity ${ }^{37}$ and to eliminate oxygenic phototrophs. Spiking was performed either at the start of the experiment or as needed based on observations of oxygenic phototroph growth. Bottles were incubated at $10^{\circ} \mathrm{C}$ under white light $\left(20-30 \mu \mathrm{mol}\right.$ photons m $\mathrm{m}^{-2}$ at 400-700 nm wavelengths; blend of incandescent and fluorescent sources) or at $22^{\circ} \mathrm{C}$ under far red LED light

330 (using a PARSource PowerPAR LED bulb; LED Grow Lights Depot; Portland, Oregon, U.S.A.). Cultures were monitored regularly for ferrous iron concentration using the ferrozine assay ${ }^{38}$ and were amended with additional freshwater medium or ferrous chloride (e.g., in 0.1-1 mM increments) when ferrous iron levels dropped, presumably due to iron oxidation.

The " $\mathrm{Ca}$. Chx. allophototropha" enrichment culture was gradually acclimated to higher concentrations of

335 freshwater medium through repeated feeding and subculture. Cultures were moved to room temperature (20$22^{\circ} \mathrm{C}$ ) growth and could be grown under white light (fluorescent, halogen, or incandescent; same light intensity as above) or under far red LED light (using a PARSource PowerPAR LED bulb). A deep agar dilution series ${ }^{39}$ was used to eliminate contaminants from the culture. The same freshwater medium was used for this dilution series, amended with an acetate feeding solution ${ }^{40}$. Ferrous chloride-containing agar plugs $(10 \mathrm{mM}$ concentration) were initially used at the bottom of tubes to form a ferrous iron concentration gradient to qualitatively determine best conditions for growth, with $0.125 \mathrm{~g} / \mathrm{L}$ sodium thioglycolate being used as a reducing agent in the medium. Over multiple rounds of optimization, the growth medium was adjusted to contain $2 \mathrm{mM}$ ferrous chloride (without sodium thioglycolate), $1.2 \mathrm{mM}$ sodium acetate, and $50 \mathrm{mg} / \mathrm{L}$ resazurin, and to be a 1:1 dilution of the original freshwater medium, kept at a $\mathrm{pH}$ of $\sim 7.5$. In addition, cultures were generally incubated 
345 in soft agar $(0.2-0.3 \% ; \mathrm{w} / \mathrm{v})^{29}$ to promote faster growth of " $\mathrm{Ca}$. Chx. allophototropha" cells compared to growth in standard concentration agar $(\sim 0.8 \% ; \mathrm{w} / \mathrm{v})$ or in liquid medium.

Agar shake tubes were incubated under $740 \mathrm{~nm}$ LED lights for a period of time to eliminate purple phototrophic bacteria, because $740 \mathrm{~nm}$ light is not readily used for photosynthesis by these bacteria ${ }^{17}$. Additionally, a higher medium $\mathrm{pH}$ of $\sim 7.5-8.5$ was used for a time to select against green phototrophic bacteria

350 belonging to the Chlorobia class, because these bacteria are known to grow poorly in moderately basic conditions ${ }^{41}$. Lastly, sodium molybdate was added to the medium at a final concentration of $100 \mu \mathrm{M}$, in a 1:10 concentration ratio relative to sulfate, to inhibit the activity of sulfate-reducing bacteria as optimized experimentally (Supplementary Methods).

\section{Physiological characterization}

Filaments of " $\mathrm{Ca}$. Chx. allophototropha" cells were identified based on pigment autofluorescence using epifluorescence microscopy. An Eclipse 600 light microscope equipped for fluorescence microscopy (Nikon; Shinagawa, Tokyo, Japan) was used with a xenon light source. Autofluorescence in the wavelength range expected for bacteriochlorphyll $c$ was detected using an excitation filter of $445( \pm 45) \mathrm{nm}$, dichroic mirror of 520 $\mathrm{nm}$, and emission filter of 715 (LP) nm (all from Semrock; Rochester, New York, USA), before light collection using an ORCA-Flash4.0 monochromatic camera (Hamamatsu Photonics; Hamamatsu City, Shizuoka, Japan). Examination of cells under epifluorescence microscopy enabled the growth of cells to be qualitatively assessed. In addition, growth of “ $\mathrm{Ca}$. Chx. allophototropha” was confirmed based on relative increases in cell pigmentation using a custom-built pigment fluorescence detection system (Supplementary Methods).

To perform transmission electron microscopy (TEM), cell biomass was picked from agar shake tubes, and residual agar surrounding cells was digested using agarase. One unit of $\beta$-agarase I (New England Biolabs; Ipswich, Massachusetts, USA) and $10 \mathrm{uL}$ of $10 \mathrm{x}$ reaction buffer was added to $100 \mu \mathrm{L}$ of cell suspension and incubated at $42^{\circ} \mathrm{C}$ for $1.5 \mathrm{~h}$. Following cell pelleting and removal of supernatant, cells were then fixed for $2 \mathrm{~h}$ at $4^{\circ} \mathrm{C}$ in $4 \% / 4 \%$ glutaraldehyde/paraformaldehyde (dissolved in 1x phosphate-buffered saline; $1 \mathrm{x}$ PBS) and stored

370 at $4^{\circ} \mathrm{C}$. Sample preparation and imaging was performed at the Molecular and Cellular Imaging Facility of the Advanced Analysis Center (University of Guelph, Ontario, Canada). Washed cell pellets were enrobed in 4\% Noble agar (ThermoFisher Scientific; Waltham, Massachusetts, USA), and enrobed samples were cut into $1 \mathrm{~mm}$ cubes. The cubes were fixed in $1 \%(\mathrm{w} / \mathrm{v})$ osmium tetroxide for $45 \mathrm{~min}$ and subjected to a $25-100 \%$ ethanol dehydration series. Samples were then subjected to stepwise infiltration using 25-100\% LR White Resin (Ted

375 Pella; Redding, California, USA), transferred to gelatin capsules in 100\% LR White Resin, and allowed to polymerize overnight at $60^{\circ} \mathrm{C}$. An Ultracut UCT ultramicrotome (Leica Microsystems; Wetzlar, Germany) equipped with a diamond knife (DiATOME; Hatfield, Pennsylvania, USA) was used to cut $50 \mathrm{~nm}$ thin sections, which were then floated on 100-mesh copper grids and stained with $2 \%$ uranyl acetate and Reynold's lead citrate to enhance contrast. Sections were imaged under standard operating conditions using a Tecnai G2 F20 TEM 
380 (ThermoFisher Scientific; Waltham, Massachusetts, USA) that was running at $200 \mathrm{kV}$ and was equipped with a Gatan 4k CCD camera (Gatan; Pleasanton, California, USA). For scanning electron microscopy (SEM), fixed cells were washed in 1x PBS and incubated in 1\% (w/v) osmium tetroxide at room temperature for $30 \mathrm{~min}$. Following incubation, cells were washed, deposited on an aluminum stub, dried, and then sputter coated with a gold:palladium mixture using a Desk V TSC Sample Preparation System (Denton Vacuum; Moorestown, New

385 Jersey, USA). Prepared samples were imaged using a Quanta FEG 250 SEM (ThermoFisher Scientific) with a high-voltage setting of $10 \mathrm{kV}$ and working distance of $9.9 \mathrm{~mm}$.

Pigments were extracted from the " $\mathrm{Ca}$. Chx. allophototropha" enrichment culture in acetone:methanol $(7: 2 \mathrm{v} / \mathrm{v})$. Cells were picked from agar shake tubes and washed once using $10 \mathrm{mM}$ Tris- $\mathrm{HCl}(\mathrm{pH}=8)$. The resulting pelleted cell material was suspended in $400 \mu \mathrm{L}$ of a solution of 7:2 acetone:methanol and was mixed

390 vigorously by vortex and pestle. Supernatant was evaporated until mostly dry and resuspended in $600 \mu \mathrm{L}$ acetone. The absorbance of the extracted pigments was assessed between 350-1000 nm using a UV-1800 UV/Visible Scanning Spectrophotometer (Shimadzu; Kyoto, Japan) and a $1 \mathrm{~mL}$ quartz cuvette.

\section{S rRNA gene amplicon sequencing and analysis}

395 The microbial community composition of early enrichment cultures was assessed using 16S ribosomal RNA (16S rRNA) gene amplicon sequencing with subsequent analysis of the obtained sequences. Genomic DNA was extracted from pelleted cell biomass using the DNeasy UltraClean Microbial Kit (Qiagen; Venlo, The Netherlands) according to the manufacturer protocol, with the addition of a 10 min treatment at $70^{\circ} \mathrm{C}$ after adding Solution SL for improved cell lysis. The V4-V5 hypervariable region of the 16S rRNA gene was then amplified from extracted DNA using the universal prokaryotic PCR primers $515 \mathrm{~F}-\mathrm{Y}^{42}$ and $926 \mathrm{R}^{43}$, using primers with attached index sequences and sequencing adaptors as described previously ${ }^{44-46}$. Amplification was performed in singlicate. Library pooling, cleanup, and sequencing on a MiSeq (Illumina; San Diego, California, USA) was performed as described previously ${ }^{44}$. Sequence data analysis was performed using QIIME2 ${ }^{47}$ version 2019.10 via the AXIOME3 ${ }^{48}$ pipeline, commit 1ec1ea6 (https://github.com/neufeld/axiome3), with default

405 analysis parameters. Briefly, paired-end reads were trimmed, merged, and denoised using DADA2 ${ }^{49}$ to generate an amplicon sequence variant (ASV) table. Taxonomic classification of ASVs was performed using QIIME2's naive Bayes classifier ${ }^{50}$ trained against the Silva SSU database ${ }^{51,52}$, release 132 . The classifier training file was prepared using QIIME2 version 2019.7.

\section{Metagenome sequencing of enrichment culture DNA}

The functional gene content of early liquid enrichment cultures was assessed via shotgun metagenome sequencing. Genomic DNA was extracted as above, and library preparation and sequencing was performed at The Centre for Applied Genomics (TCAG; The Hospital for Sick Children, Toronto, Canada). The Nextera DNA Flex Library Prep Kit (Illumina) kit was used for metagenome library preparation, and libraries were sequenced 
415 using a fraction of a lane of a HiSeq 2500 (Illumina) with 2x125 base paired-end reads to obtain 5.0-7.3 million total reads per sample.

High molecular weight DNA was later extracted from the "Ca. Chx. allophototropha" enrichment culture grown in agar shake tubes. Colonies were picked from agar, and genomic DNA was extracted using a custom phenol:chloroform DNA extraction protocol followed by size selection and cleanup (Supplementary Methods).

420 Read cloud DNA sequencing library preparation was performed using the TELL-Seq WGS Library Prep Kit ${ }^{53}$ (Universal Sequencing Technology; Canton, Massachusetts, USA) following ultralow input protocol recommendations for small genomes. Library amplification was performed using $10 \mu \mathrm{L}$ TELL-beads and 16 amplification cycles. The resulting library was sequenced using a MiSeq Reagent Kit v2 (300-cycle; Illumina) with $2 \times 150$ bp read length on a MiSeq (Illumina) to a depth of 19.6 million total reads.

\section{Genome assembly and binning}

Read cloud metagenome sequencing data for the " $\mathrm{Ca}$. Chx. allophototropha" enrichment culture were demultiplexed and quality control was performed on index reads using the Tell-Read pipeline, version 0.9.7 (Universal Sequencing Technology), with default settings. Demultiplexed reads were then assembled using Tell-

430 Link, version 1.0.0 (Universal Sequencing Technology), using global and local kmer lengths of 65 and 35, respectively. Metagenome data for this sample, together with metagenome data for an early enrichment of the same culture, were then partitioned into genome bins and analyzed using the ATLAS pipeline, version 2.2.0 ${ }^{54}$ (Supplementary Methods). The " $\mathrm{Ca}$. Chx. allophototropha” genome bin was identified based on its taxonomic placement within the Chloroflexota phylum according to the Genome Taxonomy Database Toolkit (GTDB-Tk)

435 version 0.3 .3 , which relied on GTDB release $89^{55,56}$. This genome bin was manually curated to remove potentially incorrectly binned scaffolds (Supplementary Methods). Following curation, the genome bin was annotated using Prokka version $1.14 .6^{57}$, and selected portions of the genome were checked for functional genes using the BlastKOALA webserver version $2.2^{58}$.

Short read metagenome sequencing data generated from a second early enrichment culture was also analyzed. This second enrichment was lost early in the cultivation process but contained another member of the "Ca. Chloroheliales" (Supplementary Note 1). The single enrichment culture metagenome was processed using ATLAS version 2.2.0 $0^{54,59}$ for read quality control, assembly and genome binning. A single genome bin was classified using the GTDB-Tk ${ }^{56}$ to the Chloroflexota phylum. This genome bin, named " $\mathrm{Ca}$. Chloroheliaceae bin L227-5C", was manually curated (Supplementary Methods) and was then annotated using Prokka ${ }^{57}$ as above. All

445 ATLAS settings for both runs are available in the code repository associated with this work.

To assess the community composition of the enrichment cultures, the relative abundances of each recovered genome bin from ATLAS were assessed by calculating the number of reads mapped to each bin divided by the total reads mapped to assembled scaffolds. To cross-validate these results, short protein fragments were predicted directly from unassembled read data (R1, or forward reads) using FragGeneScanPlusPlus ${ }^{60}$ 
commit 9a203d8. These protein fragments were searched using a profile Hidden Markov Model (HMM) for the single-copy housekeeping gene $r p o B$, and hits were assigned taxonomy using MetAnnotate ${ }^{61}$ version 0.92 with default settings. The HMM for rрoB (2009 release) was downloaded from FunGene ${ }^{62}$, and taxonomic classification was performed using the RefSeq database, release 80 (March 2017) ${ }^{63}$. Output of MetAnnotate was analyzed using the metannoviz R library, version 1.0.2 (https://github.com/metannotate/metannoviz) with an evalue cutoff of $10^{-10}$.

\section{Identification of RCI-associated genes}

To detect remote homologs of RCI-associated genes in the two " $\mathrm{Ca}$. Chloroheliales"-associated genome bins, hmmsearch ${ }^{64}$ version $3.1 \mathrm{~b} 2$ was used to search predicted proteins from the bins using HMMs downloaded

460 from Pfam $^{65}$. In particular, genes encoding the core Type I reaction center ( $p s c A / p s h A / p s a A B$; PF00223), a Type I reaction center-associated protein (Chlorobia-associated pscD; PF10657), chlorosomes structural units (csmAC; PF02043, PF11098), and a bacteriochlorophyll $a$ binding protein (fmoA; PF02327), were queried. Genes encoding an RCI-associated iron-sulfur protein ( $p s c B$; PF12838) and RCI-associated c-type cytochromes (Chlorobia-associated pscC or Heliobacterium-associated PetJ; PF10643 or PF13442) were also queried,

465 although the HMMs used for these genes were non-specific. The genomes were also confirmed to lack the pufLM genes associated with RCII using the "Photo_RC" HMM (PF00124), which targets both the pufL and pufM RCII subunits. Short peptides predicted directly from unassembled read data (see above) were also queried using the "Photo_RC" HMM. Detected homologs of RCI-associated genes in the genome bins were assessed for their alignment to known RCI-associated genes encoded by phototrophic microorganisms belonging to other

470 phyla. The tertiary structures of the detected homologs were also predicted using I-TASSER ${ }^{66}$, and phylogenetic placement was assessed (see below). Custom HMMs were also built for selected homologs. Primary sequences predicted for each gene of interest from the two genomes were aligned using Clustal Omega ${ }^{67}$ version 1.2.3, and HMMs were generated using hmmbuild ${ }^{64}$ version 3.1b2. Custom HMMs and homology models generated by ITASSER are available in the code repository associated with this work.

Assessment of genomic potential for photosynthesis within the Chloroflexota phylum

Representative genomes for the phylum Chloroflexota were downloaded from NCBI based on information in the GTDB ${ }^{55}$, release 89. All genomes that represented a type species of Chloroflexota according to NCBI and the GTDB were downloaded, as well as genomes representing members of known phototrophic clades (i.e., the Chloroflexaceae family ${ }^{14}$, the "Ca. Thermofonsia" $\operatorname{order}^{30}$, and the " $\mathrm{Ca}$. Roseiliniales" order ${ }^{68}$ ). Selected genomes of known phototrophs that were deposited in other genome databases were also downloaded (see details in the code repository associated with this work). In addition, any genome bins listed in the GTDB that belonged to the "54-19" order (which represented the name of the " $\mathrm{Ca}$. Chloroheliales" order in this database) were downloaded. Non-phototrophic lineages were subsequently pruned to one representative per genus, with 
485 the exception of non-phototrophic lineages closely related to phototrophic clades. In total, this left 58 genomes, including genomes of 28 known phototrophs. A concatenated core protein phylogeny was constructed for this genome collection using GToTree ${ }^{69}$ version 1.4.11. The "Bacteria.hmm" collection of 74 single-copy marker genes was used, and a minimum of $30 \%$ of the marker genes were required in each genome for the genome to be kept in the final phylogeny. IQ-TREE ${ }^{70}$ version 1.6.9 was used to build the phylogeny from the masked and

490 concatenated multiple sequence alignment. ModelFinder ${ }^{71}$ was used to determine the optimal evolutionary model for phylogeny building. Branch supports were calculated using 1000 ultrafast bootstraps ${ }^{72}$.

A collection of photosynthesis-associated genes, including genes for reaction centers, antenna proteins, chlorosome structure and attachment, bacteriochlorophyll synthesis, and carbon fixation, was selected based on the genome analyses of Tang and colleagues ${ }^{23}$ and Bryant and colleagues ${ }^{12}$. Reference sequences for these genes 495 were selected from genomes of well-studied representatives of the phylum, Chloroflexus aurantiacus ${ }^{13}$, Oscillochloris trichoides ${ }^{73}$, and Roseiflexus castenholzii ${ }^{74}$. Bidirectional BLASTP ${ }^{75}$ was performed on these reference sequences against the entire Chloroflexota genome collection (above) to detect potential orthologs. The BackBLAST pipeline ${ }^{76}$, version 2.0.0-alpha3 (doi: 10.5281/zenodo.3697265), was used for bidirectional BLASTP, and cutoffs for the $e$ value, percent identity, and query coverage of hits were empirically optimized to

$50010^{-3}, 20 \%$, and $50 \%$, respectively. Genes involved in iron oxidation or reduction were also searched using FeGenie $^{77}$, commit $30174 \mathrm{bb}$, with default settings.

\section{Phylogenetic assessment of photosynthesis-associated genes}

Selected photosynthesis-associated genes were tested against genes from other phyla to examine the 505 phylogenetic relationship of the novel " $\mathrm{Ca}$. Chloroheliales" sequences to those of other phototrophic organisms. Genes for RCI/PSI ( $p s c A / p s h A / p s a A B)^{78}$, bacteriochlorophyll $a$ binding $(f m o A)^{18}$, chlorosome structure $(c s m A)^{22}$, (bacterio)chlorophyll synthesis (bchIDH/chlIDH and $b c h L N B / \operatorname{chlLNB} / b c h X Y Z)^{12}$, and RuBisCo $(c b b L)^{79}$, were examined. Genomes potentially containing these genes of interest were determined using a combination of automated detection via AnnoTree ${ }^{80}$ and descriptions in the literature. Annotree was also used to summarize the

510 GTDB reference tree for downstream data visualization. Representative genomes from this initial genome set were selected from the GTDB, based on genome quality and taxonomic novelty, before being downloaded from NCBI. Potential orthologs of the genes of interest were identified in the downloaded genomes using bidirectional BLASTP, which was performed using the primary sequences of known reference genes as queries (Supplementary Data 2) via BackBLAST ${ }^{76}$ version 2.0.0-alpha3. In addition, for some sequence sets (i.e., Type I 515 reaction centers; Bch proteins; $\mathrm{CbbL}$ ), additional reference sequences were manually added based on literature references ${ }^{12,78,79}$. Sequence sets were then pruned to remove closely related sequences. Identified primary sequences were aligned using Clustal Omega ${ }^{67}$ version 1.2.3, and the sequence sets were further verified by manually inspecting the sequence alignments for unusual variants. Alignments were then masked from noninformative regions using Gblocks ${ }^{81}$ version $0.91 \mathrm{~b}$ with relaxed settings $(-\mathrm{t}=\mathrm{p}-\mathrm{b} 3=40-\mathrm{b} 4=4-\mathrm{b} 5=\mathrm{h})$ to preserve 
520 regions with remote homology (see results in Extended Data Table 2). Maximum likelihood protein phylogenies were built from masked sequence alignments using IQ-TREE ${ }^{70}$ version 1.6.9. Evolutionary rate model selection was performed using ModelFinder ${ }^{71}$, and 1000 rapid bootstraps were used to calculate branch support values ${ }^{72}$. Rate models used are summarized in Extended Data Table 2.

\section{Boreal Shield lake survey}

To probe the environmental relevance of "Ca . Chloroheliales" members, we surveyed eight lakes (Lakes $221,222,224,227,304,373,442$, and 626) within the IISD-ELA that were known or suspected to develop seasonal anoxia based on previous field data, along with a permanently oxic reference lake (Lake 239). These lakes were sampled for DNA in the summer or fall from 2016 until 2018 across four main sampling events.

530 Sampling in June 2016, September 2016, and September 2017 involved multi-depth hypolimnion profiling of Lakes 227 and 442, for which metagenome data have been reported previously ${ }^{82}$, along with mid-hypolimnion sampling of the other selected lakes. A full water column profile of DNA samples was collected for Lake 227 in September 2017, along with collection of RNA samples from a single depth of Lake 227 in the upper anoxic hypolimnion. Lastly, in July 2018, Lakes 221 and 304 were surveyed again. A single depth in the mid anoxic hypolimnion of both lakes was sampled for both DNA and RNA.

Samples for water column DNA were collected by pumping water, using a closed system gear pump and line, through sterile $0.22 \mu \mathrm{m}$ Sterivex polyvinyl fluoride filters (Merck Millipore; Burlington, Massachusetts, U.S.A.). The sampling line was pre-flushed with water at the desired depth prior to sampling to allow sufficient flow time for water to be fully replaced twice through the line. When collecting water column RNA, the same

540 filters were used as for DNA, but filters were immediately filled with $1.8 \mathrm{~mL}$ of DNA/RNA Shield (Zymo Research; Irvine, California, U.S.A.) once packed and purged of residual water. Filters were collected (and subsequently extracted and analyzed) in triplicate for RNA, with each filter replicate representing a distinct water column sample. Sterivex filters were kept chilled after collection until being frozen $\left(\right.$ at $\left.-20^{\circ} \mathrm{C}\right)$ upon return to the sampling camp later that day. Filters were then shipped chilled to the University of Waterloo and were kept

545 frozen $\left(\right.$ at $\left.-20^{\circ} \mathrm{C}\right)$ until processing.

\section{Environmental DNA/RNA extraction and sequencing}

For each Sterivex filter used for DNA collection, the Sterivex filter case was opened, and the filter membrane was carefully removed using a flame-sterilized scalpel blade. Each filter membrane was cut in half lengthwise along the filter; one half was stored frozen to provide a backup sample, and DNA was extracted from the other half using the DNeasy PowerSoil or DNeasy PowerSoil HTP 96 Kit (Qiagen; Venlo, The Netherlands). Extractions were performed according to the kit protocol, and the optional $10 \mathrm{~min}$ incubation at $70^{\circ} \mathrm{C}$ after adding Solution C1 was performed to enhance cell lysis. Mechanical lysis was performed for samples in PowerBead Tubes using a FastPrep-24 instrument (MP Biomedicals; Santa Ana, California, U.S.A.) set at 5 m/s 
555 for $45 \mathrm{~s}$, and mechanical lysis was performed for samples in PowerBead DNA Plates using a mixer mill MM 400 (Retsch; Haan, Germany) set at $30 \mathrm{~Hz}$ for $10 \mathrm{~min}$. Resulting DNA concentrations were then quantified using a Nanodrop spectrophotometer (Thermo Fisher Scientific; Waltham, Massachusetts, U.S.A.) or using the Qubit dsDNA HS Assay Kit with Qubit 2.0 fluorometer (Thermo Fisher Scientific).

RNA extraction was performed using the ZymoBIOMICS DNA/RNA Miniprep Kit (Zymo Research) with

560 initial steps of the protocol modified slightly to accommodate the volume of DNA/RNA Shield associated with each filter. Sterivex filters filled with DNA/RNA Shield were thawed, and the DNA/RNA Shield was pumped out of the filter cases and saved for downstream use. Filter cases were then opened and filters excised as described above. Each filter half was cut into small pieces. One half was stored in a clean $2 \mathrm{~mL}$ microfuge tube along with half of the collected DNA/RNA Shield solution and frozen as a backup sample. The other filter half, 565 along with the remaining DNA/RNA Shield, was added into a dry ZR BashingBead Lysis Tube for extraction. Mechanical lysis was performed using a FastPrep-24 instrument (MP Biomedicals). Lysis tubes were shaken at $6.5 \mathrm{~m} / \mathrm{s}$ for $60 \mathrm{~s}$ twice, and tubes were allowed to cool on ice for at least $60 \mathrm{~s}$ between mechanical lysis rounds. After centrifugation, the entire supernatant volume was transferred to a new microfuge tube, and one volume of DNA/RNA Lysis Buffer was added to the tube and mixed. RNA extraction was then performed according to the 570 "DNA \& RNA Parallel Purification" protocol in the kit manual using in-column DNase I treatment. Only RNA (and not DNA) extracts were saved because corresponding Sterivex filters for DNA were collected and processed for the same lake depths using the protocol described above. The resulting RNA extracts were quantified using a Nanodrop spectrophotometer (Thermo Fisher Scientific) and Qubit RNA Assay Kit with Qubit 2.0 fluorometer (Thermo Fisher Scientific). Extracts were also run on a 1\% agarose gel stained with GelRed

575 (Biotium; Fremont, California, U.S.A.) to confirm that rRNA of the expected lengths was visible.

Metagenomes were sequenced in two separate batches. Lake hypolimnion DNA samples from the June and September 2016 sampling events were prepared into sequencing libraries and sequenced by the U.S. Department of Energy Joint Genome Institute (Lawrence Berkeley National Laboratory) . Metagenome library preparation was performed using the Nextera XT DNA Library Preparation Kit including library amplification steps. Resulting libraries were sequenced on a HiSeq 2500 (Illumina) with 2x150 base paired end reads, generating 88.2 to 273.5 million reads per sample. Subsequently, DNA samples from 2017 and 2018 field sampling were prepared for metagenome sequencing and sequenced by the McMaster Genome Facility (McMaster University; Hamilton, Ontario, Canada). Sample DNA was sheared to the desired insert size using a Covaris ultrasonicator (Covaris; Woburn, Massachusetts, U.S.A.), and metagenome sequencing libraries were

585 prepared using the NEBNext Ultra II DNA Library Prep Kit for Illumina (New England Biolabs) including library amplification steps. The resulting library was sequenced on a portion of a lane of a HiSeq 2500 with $2 \times 200$ base paired end reads. Because the reverse reads for that run were truncated at $114 \mathrm{bp}$ due to a sequencer error, the library was run a second time on a single lane of a HiSeq 2500 with 2x250 base paired end reads. Data was combined between the two runs, generating a total of 41.2 to 128.2 million reads per sample. 

library preparation and sequencing was performed by the McMaster Genome Facility (McMaster University). The Ribo-Zero rRNA Removal Kit (Bacteria; Illumina) was used to deplete rRNA genes in the samples prior to library preparation. Library preparation was then performed using the NEBNext Ultra II RNA Library Prep Kit for Illumina (New England Biolabs). Consistent RNA amounts for all samples, following rRNA depletion, were used as input and without directional RNA selection. The resulting library was sequenced on a portion of a lane of a HiSeq 2500 in Rapid Run Mode with 2x200 bp paired end reads. This was the same sequencing run as for 2017-2018 metagenomes where the reverse reads were truncated at $114 \mathrm{bp}$, but because sufficient sequencing depth was obtained for the project's research goal, the library was not re-run. Sequencing generated 11.1 to 20.5 million reads per replicate. In total, 37 metagenomes were sequenced, along with nine metatranscriptomes representing three samples (see Supplementary Data 3-4).

\section{Metagenome and metatranscriptome analysis}

All metagenome data was processed using the ATLAS pipeline, version 2.1.4 ${ }^{54}$. Default settings were used for the run except that the minimum percent identity threshold for read mapping (via "contig_min_id") was set

605 to $99 \%$ and only MaxBin2 and MetaBAT2 were used as binning algorithms ${ }^{83,84}$. To enhance genome binning quality, six lake metagenomes that were previously sequenced from the water columns of Lakes 227 and $442^{82}$ were included in the ATLAS run, along with a single metagenome from the aphotic zone of the nearby and meromictic Lake 111, which was also sampled in July 2018. The Lake 111 metagenome was not analyzed further in the context of this work. The entire ATLAS pipeline, including quality control on raw reads, metagenome

610 assembly of individual samples, metagenome binning, dereplication of bins from all samples and bin analysis, and gene clustering and annotation, was run end-to-end. For the genome binning step, all metagenome samples from the same lake were summarized in the same "BinGroup", allowing for read mapping information between each sample from the same lake to be used as differential abundance information to guide genome binning. After running ATLAS, the resulting dereplicated genome bins were taxonomically classified using the GTDB-Tk,

615 version $0.3 .2^{56}$, which relied on the GTDB, release $89^{55}$. All metagenome-assembled genomes (MAGs) had a minimum percent completeness of $50 \%$ and maximum percent contamination of $10 \%$ based on $\mathrm{CheckM}^{85}$, according to default ATLAS settings. The relative abundance of each MAG in a sample was calculated by dividing the number of QC-processed reads mapped to the MAG by the total number of assembled reads (i.e., raw reads that mapped to assembled contigs) for that sample. A MAG was considered present in a sample if its 620 relative abundance was greater than $0.01 \%$.

Metatranscriptome data were also processed using the ATLAS pipeline ${ }^{54}$. Using ATLAS commit 59da38f, the "qc" module was run to perform quality control of raw metatranscriptome read data. Then, a customized fork of ATLAS, commit 96e47df (available under the "maprna" branch at https://github.com/jmtsuji/atlas) was used to map RNA reads onto the set of dereplicated genome bins obtained from metagenome analyses (above) and to 
summarize RNA read counts. Briefly, the dereplicated genome bins were used as input for the "genomes" module of ATLAS so that QC-processed metatranscriptome reads were mapped onto genome bins using bbmap. The minimum percent identity threshold for read mapping (“contig_min_id") was set to 99\%. Following read mapping to genome bins, the counts of metatranscriptome read hits to genes within genome bins were summarized using featureCounts ${ }^{86}$, in version 1.6.4 of the Subread package. Default settings were used, except

630 for the following flags: “-t CDS -g ID --donotsort”. Raw analysis code is available in the Github repository associated with this work.

After generating RNA read mapping data via ATLAS, the relative expression of each dereplicated genome bin was calculated within each metatranscriptome. To perform this calculation, within each metatranscriptome, the number of RNA reads that mapped to each genome bin was divided by the total number of RNA reads that

635 mapped to all genome bins. The resulting relative expression values were averaged between replicate metatranscriptomes. In addition, the expression levels of individual genes within a genome were determined for "Ca. Chloroheliales"-associated genome bins, which were identified based on GTDB taxonomy. The single-copy taxonomic marker gene $d n a K$ was identified in each genome bin based on eggNOG-mapper ${ }^{87}$ annotations (version 1.0.3), and the annotation was confirmed based on a BLASTP ${ }^{75}$ search of the predicted protein sequence

640 against the RefSeq ${ }^{63}$ database. Relative expression levels of each gene within a genome bin were then calculated by dividing the gene length-normalized hit count of each gene by the gene-length normalized hit count of $d n a K$. Annotations of genes with high relative expression were determined by eggNOG-mapper as part of the ATLAS "Genecatalog" module, run on metagenome data, combined with manual searching for phototrophy-associated genes using methods described above.

645

\section{Data availability}

The three enrichment culture metagenomes, along with recovered genome bins, are available under the National Center for Biotechnology Information (NCBI) BioProject accession PRJNA640240. Amplicon sequencing data are available under the same NCBI BioProject accession. Metagenome data from 2016, 650 sequenced by the JGI, are available in the JGI Genome Portal under Proposal ID 502896. Environmental metagenome and metatranscriptome data from 2017-2018 are available under NCBI BioProject accession PRJNA664486. The full set of 756 metagenome-assembled genomes used for read mapping of metatranscriptome data are available in a Zenodo repository (doi: $10.5281 /$ zenodo.3930110) and will be made available on NCBI upon publication.

\section{Code availability}

Custom scripts and additional raw data files used to analyze the metagenome and genome data are available at https://github.com/jmtsuji/Ca-Chlorohelix-allophototropha-RCI (doi: 10.5281/zenodo.3932366). 
660

43. Quince, C., Lanzen, A., Davenport, R. J. \& Turnbaugh, P. J. Removing noise from pyrosequenced amplicons. BMC Bioinform 12, 38 (2011).

44. Kennedy, K., Hall, M. W., Lynch, M. D. J., Moreno-Hagelsieb, G. \& Neufeld, J. D. Evaluating bias of

45. Bartram, A. K., Lynch, M. D., Stearns, J. C., Moreno-Hagelsieb, G. \& Neufeld, J. D. Generation of multimillion-sequence 16S rRNA gene libraries from complex microbial communities by assembling paired-end Illumina reads. Appl Environ Microbiol 77, 3846-3852 (2011).

46. Cavaco, M. A. et al. Freshwater microbial community diversity in a rapidly changing High Arctic

47. Bolyen, E. et al. Reproducible, interactive, scalable and extensible microbiome data science using QIIME 2. Nat Biotechnol 37, 852-857 (2019).

48. Min, D., Doxey, A. C. \& Neufeld, J. D. AXIOME3: automation, extension, and integration of microbial ecology. GigaScience 10, giab006 (2021).

700 49. Callahan, B. J. et al. DADA2: High-resolution sample inference from Illumina amplicon data. Nat Methods 13, 581-583 (2016).

50. Bokulich, N. A. et al. Optimizing taxonomic classification of marker-gene amplicon sequences with QIIME 2's q2-feature-classifier plugin. Microbiome 6, 90 (2018).

51. Quast, C. et al. The SILVA ribosomal RNA gene database project: improved data processing and web-

$$
\text { based tools. Nucleic Acids Res 41, D590-D596 (2013). }
$$


52. Glöckner, F. O. et al. 25 years of serving the community with ribosomal RNA gene reference databases and tools. J Biotechnol 261, 169-176 (2017).

53. Chen, Z. et al. Ultra-low input single tube linked-read library method enables short-read secondgeneration sequencing systems to generate highly accurate and economical long-range sequencing information routinely. Genome Res 30, 898-909 (2020).

54. Kieser, S., Brown, J., Zdobnov, E. M., Trajkovski, M. \& McCue, L. A. ATLAS: a Snakemake workflow for assembly, annotation, and genomic binning of metagenome sequence data. BMC Bioinform 21, 257 (2020).

55. Parks, D. H. et al. A standardized bacterial taxonomy based on genome phylogeny substantially revises the tree of life. Nat Biotechnol 36, 996-1004 (2018).

56. Chaumeil, P.-A., Mussig, A. J., Hugenholtz, P. \& Parks, D. H. GTDB-Tk: a toolkit to classify genomes with the Genome Taxonomy Database. Bioinformatics 36, 1925-1927 (2020).

57. Seemann, T. Prokka: rapid prokaryotic genome annotation. Bioinformatics 30, 2068-2069 (2014).

58. Kanehisa, M., Sato, Y. \& Morishima, K. BlastKOALA and GhostKOALA: KEGG tools for functional characterization of genome and metagenome sequences. J Mol Biol 428, 726-731 (2016).

59. White III, R. A. et al. ATLAS (Automatic Tool for Local Assembly Structures) - a comprehensive infrastructure for assembly, annotation, and genomic binning of metagenomic and metatranscriptomic data. PeerJ Preprints 5, e2843v1 (2017).

60. Singh, R. G. et al. Unipept 4.0: functional analysis of metaproteome data. J Proteome Res 18, 606-615 (2018).

61. Petrenko, P., Lobb, B., Kurtz, D. A., Neufeld, J. D. \& Doxey, A. C. MetAnnotate: function-specific taxonomic profiling and comparison of metagenomes. BMC Biol 13, 1-8 (2015).

62. Fish, J. A. et al. FunGene: the functional gene pipeline and repository. Front Microbiol 4, 291 (2013).

63. O'Leary, N. A. et al. Reference sequence (RefSeq) database at NCBI: current status, taxonomic expansion, and functional annotation. Nucleic Acids Res 44, D733-D745 (2016).

64. Eddy, S. R. Accelerated profile HMM searches. PLOS Comput Biol 7, e1002195 (2011).

65. Finn, R. D. et al. The Pfam protein families database: towards a more sustainable future. Nucleic Acids Res 44, D279-D285 (2016).

66. Yang, J. et al. The I-TASSER Suite: protein structure and function prediction. Nat Methods 12, 7-8 (2015).

67. Sievers, F. et al. Fast, scalable generation of high-quality protein multiple sequence alignments using Clustal Omega. Mol Syst Biol 7, 539 (2011).

68. Tank, M., Thiel, V., Ward, D. M. \& Bryant, D. A. A panoply of phototrophs: an overview of the thermophilic chlorophototrophs of the microbial mats of alkaline siliceous hot springs in Yellowstone National Park, WY, USA. in Modern Topics in the Phototrophic Prokaryotes: Environmental and Applied Aspects (ed. Hallenbeck, P. C.) 87-137 (Springer International Publishing Switzerland, 2017). doi:10.1007/978-3-319-46261-5_3.

69. Lee, M. D. GToTree: a user-friendly workflow for phylogenomics. Bioinformatics 35, 4162-4164 (2019).

745 70. Nguyen, L.-T., Schmidt, H. A., von Haeseler, A. \& Minh, B. Q. IQ-TREE: a fast and effective stochastic algorithm for estimating maximum-likelihood phylogenies. Mol Biol Evol 32, 268-274 (2015).

71. Kalyaanamoorthy, S., Minh, B. Q., Wong, T. K. F., von Haeseler, A. \& Jermiin, L. S. ModelFinder: fast model selection for accurate phylogenetic estimates. Nat Methods 14, 587-589 (2017).

72. Hoang, D. T., Chernomor, O., von Haeseler, A., Minh, B. Q. \& Vinh, L. S. UFBoot2: improving the ultrafast bootstrap approximation. Mol Biol Evol 35, 518-522 (2018). 
73. Keppen, O. I., Baulina, O. I. \& Kondratieva, E. N. Oscillochloris trichoides neotype strain DG-6. Photosynth Res 41, 29-33 (1994).

74. Hanada, S., Takaichi, S., Matsuura, K. \& Nakamura, K. Roseiflexus castenholzii gen. nov., sp. nov., a thermophilic, filamentous, photosynthetic bacterium that lacks chlorosomes. Int J Syst Evol Microbiol 52, 187-193 (2002).

75. Altschul, S. F., Gish, W., Miller, W., Myers, E. W. \& Lipman, D. J. Basic local alignment search tool. $J$ Mol Biol 215, 403-410 (1990).

76. Bergstrand, L. H., Cardenas, E., Holert, J., Hamme, J. D. V. \& Mohn, W. W. Delineation of steroiddegrading microorganisms through comparative genomic analysis. mBio 7, e00166-16 (2016).

Garber, A. I. et al. FeGenie: A comprehensive tool for the identification of iron genes and iron gene neighborhoods in genome and metagenome assemblies. Front Microbiol 11, 37 (2020).

78. Cardona, T. Early Archean origin of heterodimeric Photosystem I. Heliyon 4, e00548 (2018).

79. Tabita, F. R., Hanson, T. E., Satagopan, S., Witte, B. H. \& Kreel, N. E. Phylogenetic and evolutionary relationships of RubisCO and the RubisCO-like proteins and the functional lessons provided by diverse molecular forms. Phil Trans R Soc B 363, 2629-2640 (2008).

80. Mendler, K. et al. AnnoTree: visualization and exploration of a functionally annotated microbial tree of life. Nucleic Acids Res 47, 4442-4448 (2019).

81. Talavera, G. \& Castresana, J. Improvement of phylogenies after removing divergent and ambiguously aligned blocks from protein sequence alignments. Syst Biol 56, 564-577 (2007).

Tsuji, J. M. et al. Anoxygenic photosynthesis and iron-sulfur metabolic potential of Chlorobia populations from seasonally anoxic Boreal Shield lakes. ISME J 14, 2732-2747 (2020).

83. Wu, Y.-W., Simmons, B. A. \& Singer, S. W. MaxBin 2.0: an automated binning algorithm to recover genomes from multiple metagenomic datasets. Bioinformatics 32, 605-607 (2016).

84. Kang, D. D. et al. MetaBAT 2: an adaptive binning algorithm for robust and efficient genome

85. Parks, D. H., Imelfort, M., Skennerton, C. T., Hugenholtz, P. \& Tyson, G. W. CheckM: assessing the quality of microbial genomes recovered from isolates, single cells, and metagenomes. Genome Res 25, 1043-1055 (2015).

86. Liao, Y., Smyth, G. K. \& Shi, W. featureCounts: an efficient general purpose program for assigning sequence reads to genomic features. Bioinformatics 30, 923-930 (2014).

87. Huerta-Cepas, J. et al. Fast genome-wide functional annotation through orthology assignment by eggNOG-mapper. Mol Biol Evol 34, 2115-2122 (2017).

\section{Acknowledgments}

With gratitude (chi-miigwech), we acknowledge that our samples were obtained from the traditional territory of the Anishinaabe Peoples. We also thank staff at the IISD-ELA and R. Elgood for providing baseline limnological data and sampling advice; J. Mead, E. Barber, J. Wolfe, K. Thompson, E. McQuay, and R. Henderson for assistance with lake sampling; X. Lu, E. Spasov, and L. Shakib, for assistance with DNA and RNA extraction; K. Engel for assistance with high-throughput DNA sequencing; Y. Shirotori and M. Saini for assistance with enrichment cultivation; V. Gaisin for advice for cultivating filamentous phototrophs; S. Hirose for assistance with Sanger sequencing and pigment analyses; R. Harris and E. Roach for assistance with electron microscopy; T. Chen for advice during read cloud sequencing library preparation; Y. Wang for assistance with read cloud sequence assembly; V. Thiel for advice about reference genomes of Chloroflexota members; B. 
Schink for advice about binomial nomenclature; N. Tran, K. Shimada, and J. Hemp for discussion and advice about biochemistry; and D. Bryant for critical reading of this work. This work was supported by a Strategic Partnership Grant for Projects from the National Sciences and Engineering Research Council of Canada (NSERC), Discovery Grants from NSERC, and a research grant from the Institute for Fermentation, Osaka. The work conducted by the U.S. Department of Energy Joint Genome Institute, a DOE Office of Science User Facility, was supported under Contract No. DE-AC02-05CH11231.

800

\section{Author contributions}

J.J.V., S.L.S., and J.D.N. conceived the study. J.M.T., N.A.S., and M.T. performed enrichment cultivation. J.M.T. collected epifluorescence microscopy images and ran pigment analyses. J.M.T. and N.A.S. performed electron microscopy, extracted culture genomic DNA, and performed read cloud metagenome library preparation

805 and sequencing. J.M.T., J.J.V., and S.L.S. performed the lake survey, and J.M.T. and N.A.S. performed environmental DNA and RNA extraction. J.M.T. analyzed 16S rRNA gene, metagenome, and metatranscriptome sequencing data, conducted phylogenetic and statistical analyses, and visualized the data. J.M.T., S.N., S.H., M.T., and J.D.N. interpreted the data and its impacts on understanding the evolution of photosynthesis. S.H., M.T., and J.D.N. supervised research. J.M.T. wrote the paper with N.A.S., S.N., J.D.N., and comments from all 810 other authors.

\section{Ethics declarations}

Competing interests

The authors declare no competing interests. 
820 Supplementary Data 1 | Genomic context of novel Type I reaction center genes detected in this study. The Excel file summarizes the top five BLASTP hits against the RefSeq database for predicted open reading frames (ORFs) of each of 20 genes up/downstream of the detected $p s c A$-like genes.

\section{Supplementary Data 2 | Bidirectional BLASTP results for photosynthesis-related genes among the}

825 Chloroflexota phylum. The Excel file summarizes the query sequences and results of bidirectional BLASTP to search for photosynthesis-related genes in genomes of Chloroflexota members. These data are visualized in Fig. 2.

Supplementary Data 3 | Mapping of metagenome reads to metagenome-assembled genomes. The Excel file 830 summarizes the percent mapped reads from each analyzed Boreal Shield lake metagenome to each of the 756 dereplicated MAG recovered in this study.

Supplementary Data 4 | Mapping of metatranscriptome reads to metagenome-assembled genomes. The Excel file summarizes the percent mapped reads from each analyzed Boreal Shield lake metatranscriptome to 835 each of the 756 dereplicated MAG recovered in this study. For each metatranscriptome, the percentage of total reads that mapped to the MAG set is also summarized. Only MAGs with at least one mapped read are shown. Data for Lakes 221 and 304 are visualized in Fig. 3a.

Supplementary Data 5 | Gene expression of "Ca. Chloroheliales"-associated genome bins. The Excel file 840 summarizes the normalized expression values of protein-coding genes in MAGs 319 and 729 based on environmental metatranscriptome data from Lakes 221 and 304. These data are visualized in Fig. 3b and Extended Data Fig. 7.

Supplementary Data 6 | Amplicon sequencing variant table of early phototroph enrichment cultures from

845 this study. The Excel file summarizes the percent relative abundances of amplicon sequencing variants (ASVs) detected in 16S ribosomal RNA gene amplicon sequencing data representing early enrichment cultures of " $\mathrm{Ca}$. Chloroheliales" members. 
bioRxiv preprint doi: https://doi.org/10.1101/2020.07.07.190934; this version posted August 7, 2021. The copyright holder for this preprint (which was not certified by peer review) is the author/funder, who has granted bioRxiv a license to display the preprint in perpetuity. It is made available under aCC-BY-NC-ND 4.0 International license.

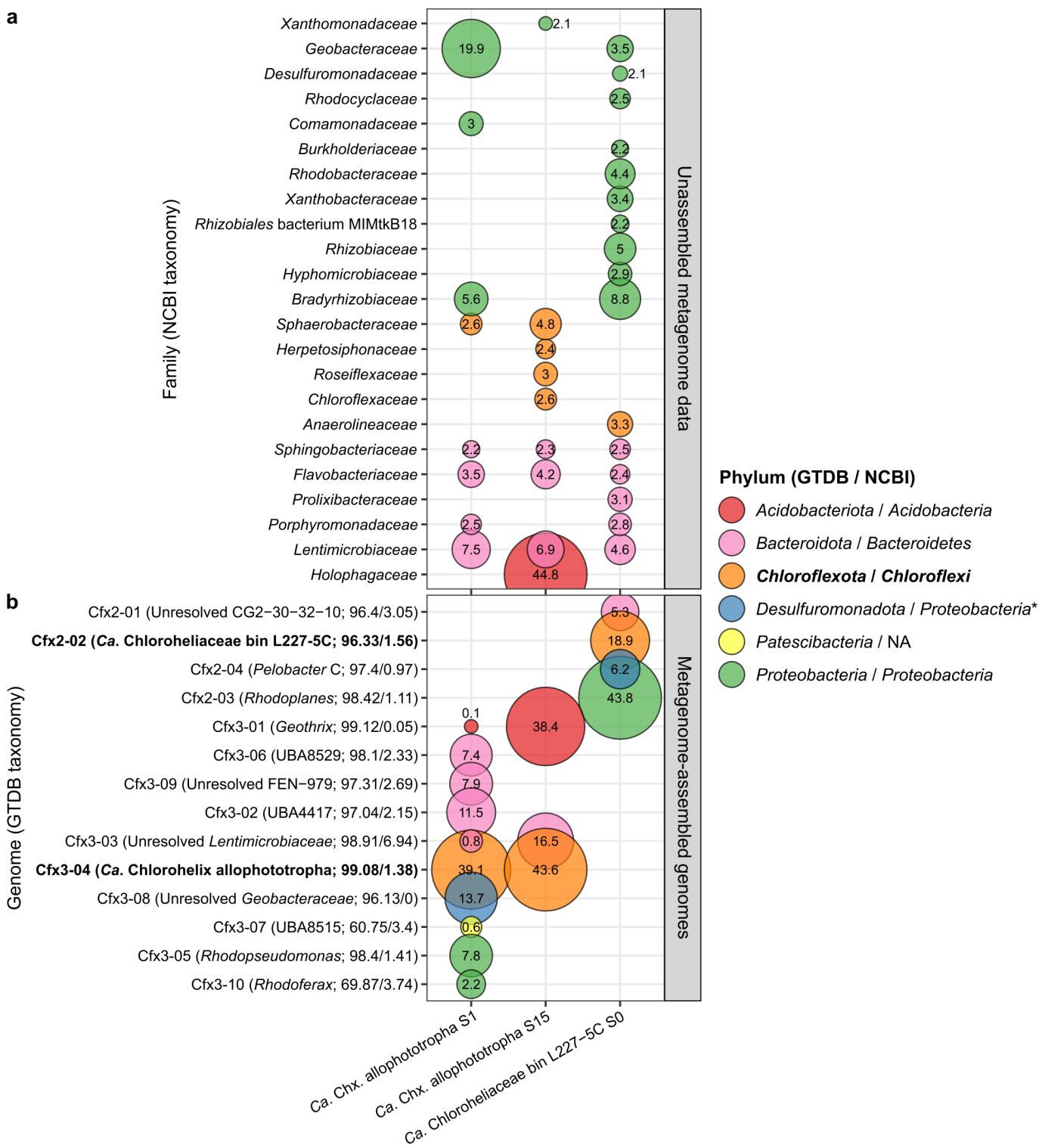

Extended Data Fig. 1 | Composition of the " $\mathrm{Ca}$. Chloroheliales" enrichment cultures described in this study. a, Bubble plot showing the relative abundances of taxa within " $\mathrm{Ca}$. Chloroheliales" enrichment culture metagenomes based on classification of partial RpoB fragments recovered from unassembled reads. Bubbles are coloured based on phylum according to NCBI taxonomy, because reads were classified against the RefSeq database. Microbial families with greater 855 than $2 \%$ relative abundance are shown. Two metagenomes were derived from subcultures \#1 and 15 of the "Ca. Chx. allophototropha" culture, and the third metagenome was derived from the initial enrichment of " $\mathrm{Ca}$. Chloroheliaceae bin L227-5C" that was eventually lost. The subculture 15 metagenome of " $\mathrm{Ca}$. Chx. allophototropha" was used to assemble the final genome of the strain. $\mathbf{b}$, Bubble plot showing the relative abundances of uncurated genome bins recovered from the same metagenomes. Relative abundances are expressed as the percentage of reads mapped to the genome bin compared to the total number of assembled reads. Bubbles are coloured by phylum according to GTDB taxonomy and are shown for all entries greater than $0.05 \%$ relative abundance. Listed beside each genome bin name are the GTDB genus that the bin was classified to and the percent completeness and contamination of the bin according to CheckM. Statistics shown for the " $\mathrm{Ca}$. Chx. allophototropha" bin differ slightly compared to Supplementary Note 2, which describes the statistics for the bin after manual bin curation. 
bioRxiv preprint doi: https://doi.org/10.1101/2020.07.07.190934; this version posted August 7, 2021. The copyright holder for this preprint (which was not certified by peer review) is the author/funder, who has granted bioRxiv a license to display the preprint in perpetuity. It is made available under aCC-BY-NC-ND 4.0 International license.


Extended Data Fig. 2 | Maximum likelihood phylogeny of oxygenic and anoxygenic Type I reaction center predicted protein sequences. A simplified depiction of the same phylogeny is shown in Fig. 1b. The phylogeny is midpoint rooted, and ultrafast bootstrap values of at least $80 / 100$ are shown. The scale bar represents the expected proportion of amino acid change across the 548-residue masked sequence alignment (Extended Data Table 2). 


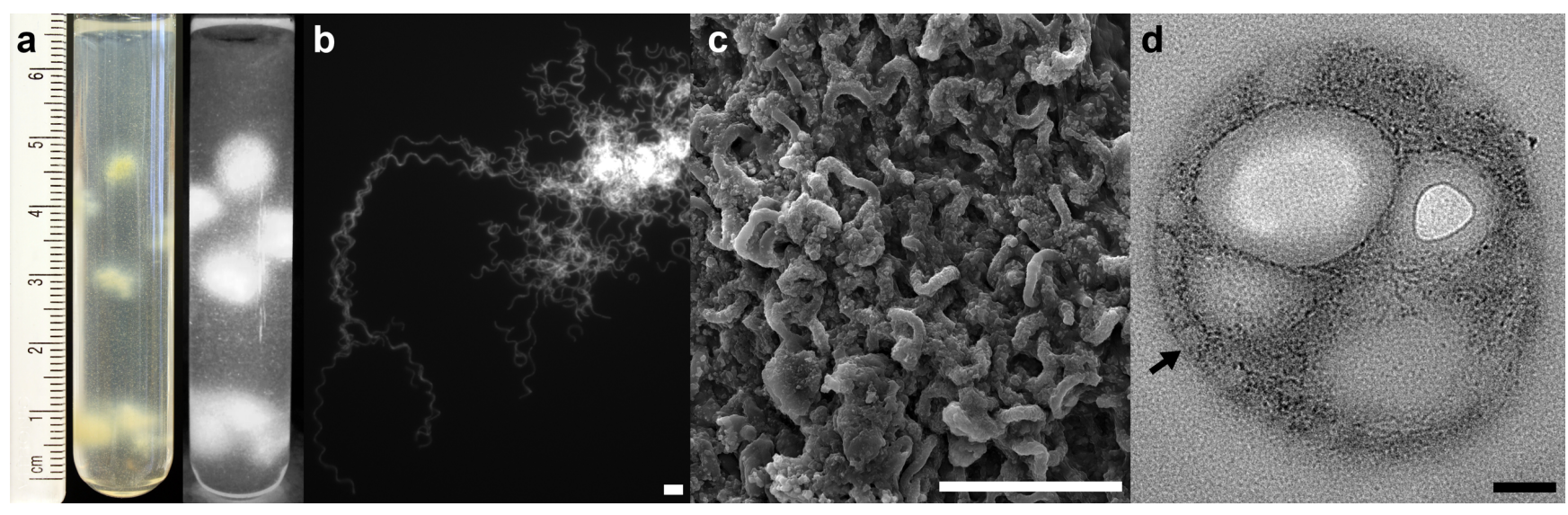

Extended Data Fig. 3 | Physiology of the "Ca. Chx. allophototropha" culture. a, Example growth of the "Ca. Chx.

875 allophototropha" enrichment in soft agar. The left panel is a photograph of a test tube with a ruler shown for scale, and the right panel shows the same tube when viewed under a pigment fluorescence detection system optimized for bacteriochlorophyll $c$ (Supplementary Methods; Extended Data Fig. 11). b, Epifluorescence microscopy image of " $\mathrm{Ca}$. Chx. allophototropha" cells. Cell autofluorescence is shown, with light excitation and detection wavelengths optimized for bacteriochlorophyll $c$. c, Scanning electron microscopy image of an aggregate of " $\mathrm{Ca}$. Chx. allophototropha" cells. d,

880 Transmission electron microscopy image showing a cross sections of " $\mathrm{Ca}$. Chx. allophototropha" cells. An example chlorosome is marked with an arrow. The scale bar in panel $\mathbf{b}$ represents $10 \mu \mathrm{m}$, and scale bars in panels $\mathbf{c}-\mathbf{d}$ represent 0.1 $\mu \mathrm{m}$. See Fig. 1 for additional microscopy images. 

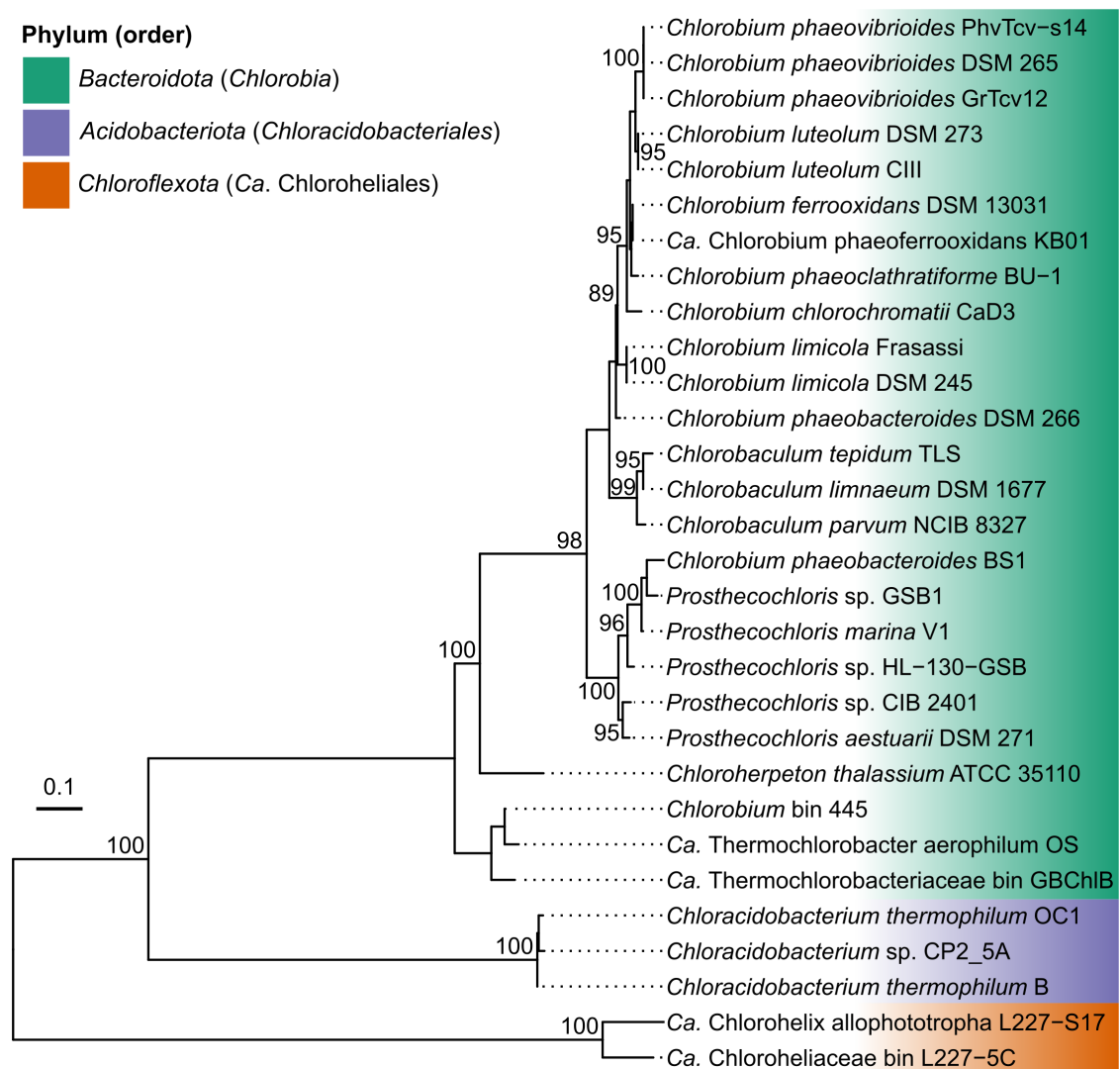

Extended Data Fig. 4 | Maximum likelihood phylogeny of Fenna-Matthews-Olson (FMO) protein (FmoA) sequences. The phylogeny is midpoint rooted, and ultrafast bootstrap values of at least $80 / 100$ are shown. The scale bar represents the expected proportion of amino acid change across the 356-residue masked sequence alignment (Extended Data Table 2). 


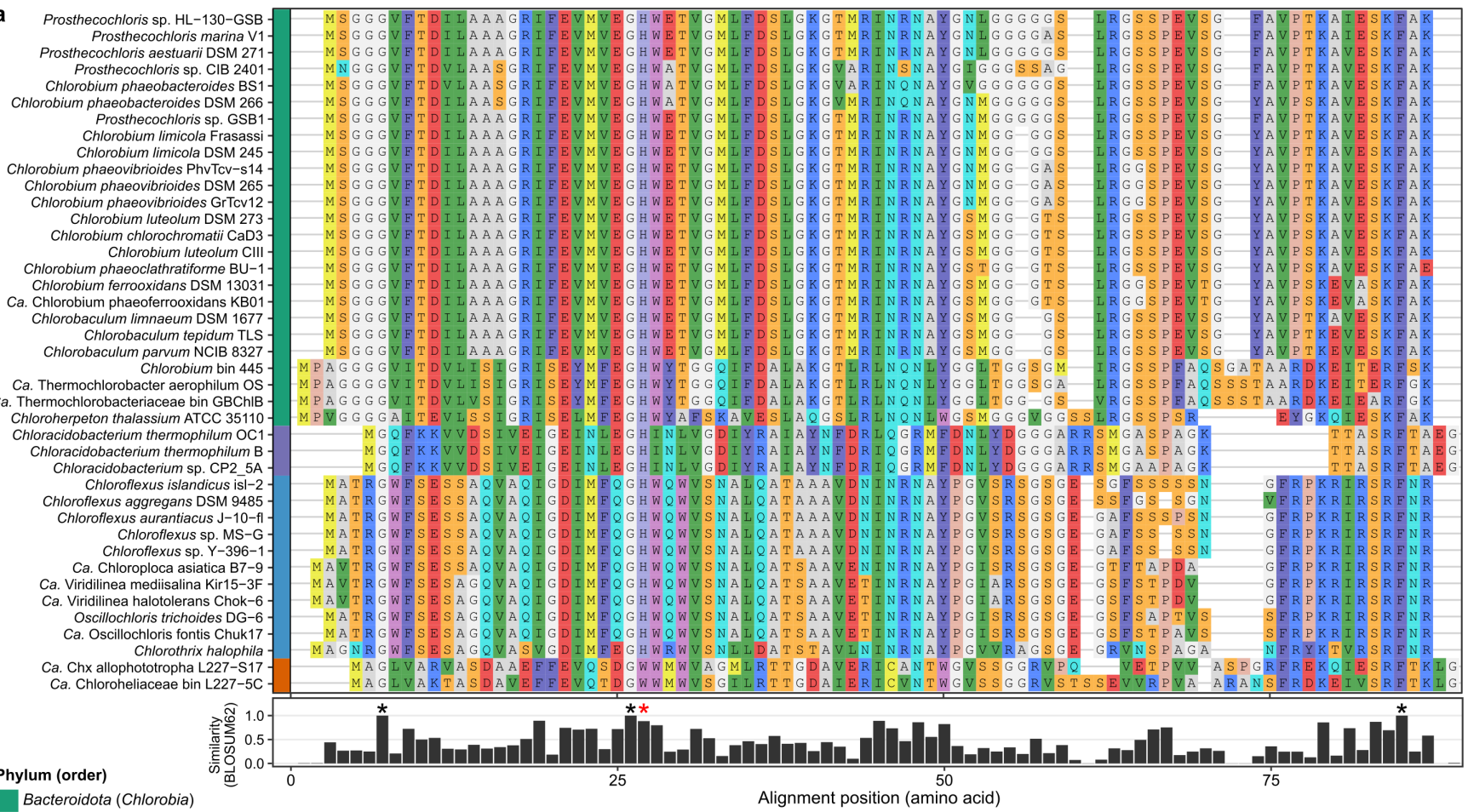

Bacteroidota (Chlorobia)
Acidobacteriota (Chloracidobacteriales)
Chloroflexota (Chloroflexales)
Chloroflexota (Ca. Chloroheliales) b

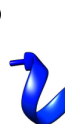

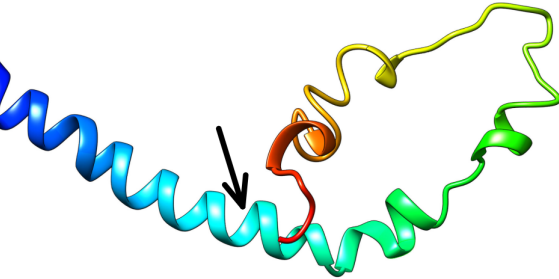

c

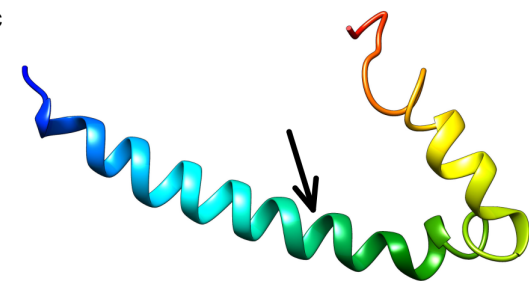

Extended Data Fig. 5 | Structural properties of the predicted chlorosome protein $\mathrm{CsmA}$ in "Ca. Chx.

allophototropha". a, Multiple sequence alignment of the CsmA primary sequence including representatives from known chlorosome-containing phyla. The entire CsmA primary sequence is shown, and a colour bar on the left of the alignment

895 indicates the phylum and order associated with each sequence. A corresponding maximum likelihood phylogeny is shown in Extended Data Fig. 8. The BLOSUM62 sequence similarity score is shown underneath the alignment. Residues that are conserved across all sequences are marked with a black asterisk, and the His 25 residue predicted to be involved in bacteriochlorophyll $a$ binding is marked with a red asterisk. b. Homology model showing the possible tertiary structure of the " $\mathrm{Ca}$. Chx. allophototropha" CsmA protein as predicted by I-TASSER. The tryptophan residue corresponding to the typical His 25 residue is indicated by an arrow. c, Tertiary structure of the CsmA protein from Chlorobium tepidum (PDB accession $2 \mathrm{~K} 37$ ); the His25 site is marked with an arrow. 
bioRxiv preprint doi: https://doi.org/10.1101/2020.07.07.190934; this version posted August 7, 2021. The copyright holder for this preprint (which was not certified by peer review) is the author/funder, who has granted bioRxiv a license to display the preprint in perpetuity. It is made available under aCC-BY-NC-ND 4.0 International license.


905 Extended Data Fig. 6 | Maximum likelihood phylogeny of RuBisCO large subunit (CbbL) predicted protein sequences. Group I to III CbbL sequences are shown, and group IV sequences, which do not form proteins involved in carbon fixation, are omitted for conciseness. The phylogeny is midpoint rooted, and ultrafast bootstrap values of at least $80 / 100$ are shown. The scale bar represents the expected proportion of amino acid change across the 412-residue masked sequence alignment (Extended Data Table 2). 
bioRxiv preprint doi: https://doi.org/10.1101/2020.07.07.190934; this version posted August 7, 2021. The copyright holder for this preprint (which was not certified by peer review) is the author/funder, who has granted bioRxiv a license to display the preprint in perpetuity. It is made available under aCC-BY-NC-ND 4.0 International license.

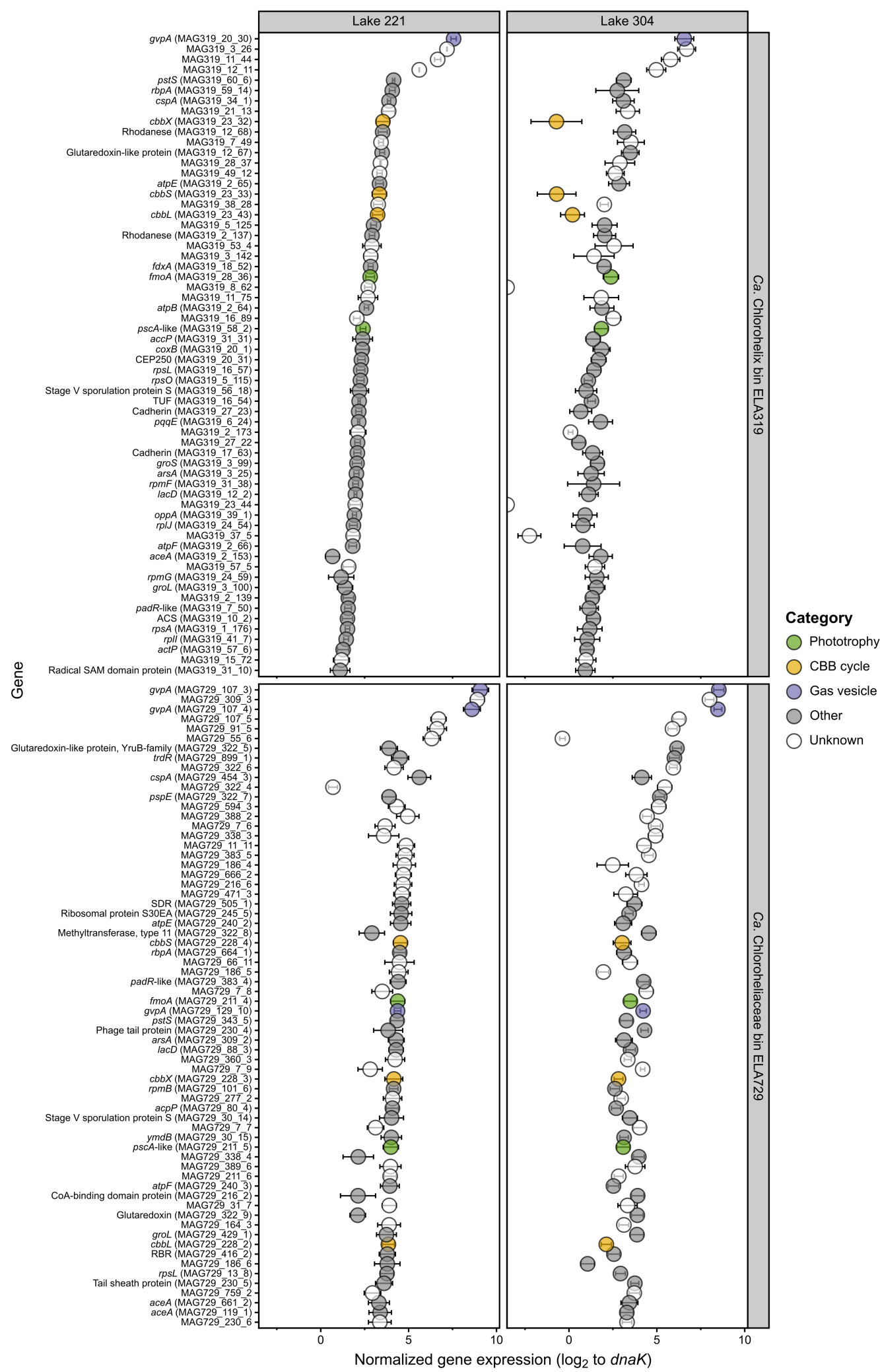

Extended Data Fig. 7 | Highly expressed genes among “ $\mathrm{Ca}$. Chloroheliales"-associated genome bins within Lake 221 and 304 metatranscriptome data. The top 50 protein-coding genes with highest normalized expression values are shown for genome bins ELA319 and 729. Error bars represent the standard deviation of the $\log _{2}$ expression ratio based on triplicate metatranscriptomes $(n=3)$. Genes potentially involved in phototrophy, carbon fixation, and buoyancy are highlighted. Normalized expression values for all genes, along with their predicted amino acid sequences and annotations, are included in Supplementary Data 5. 
bioRxiv preprint doi: https://doi.org/10.1101/2020.07.07.190934; this version posted August 7, 2021. The copyright holder for this preprint (which was not certified by peer review) is the author/funder, who has granted bioRxiv a license to display the preprint in perpetuity. It is made available under aCC-BY-NC-ND 4.0 International license.

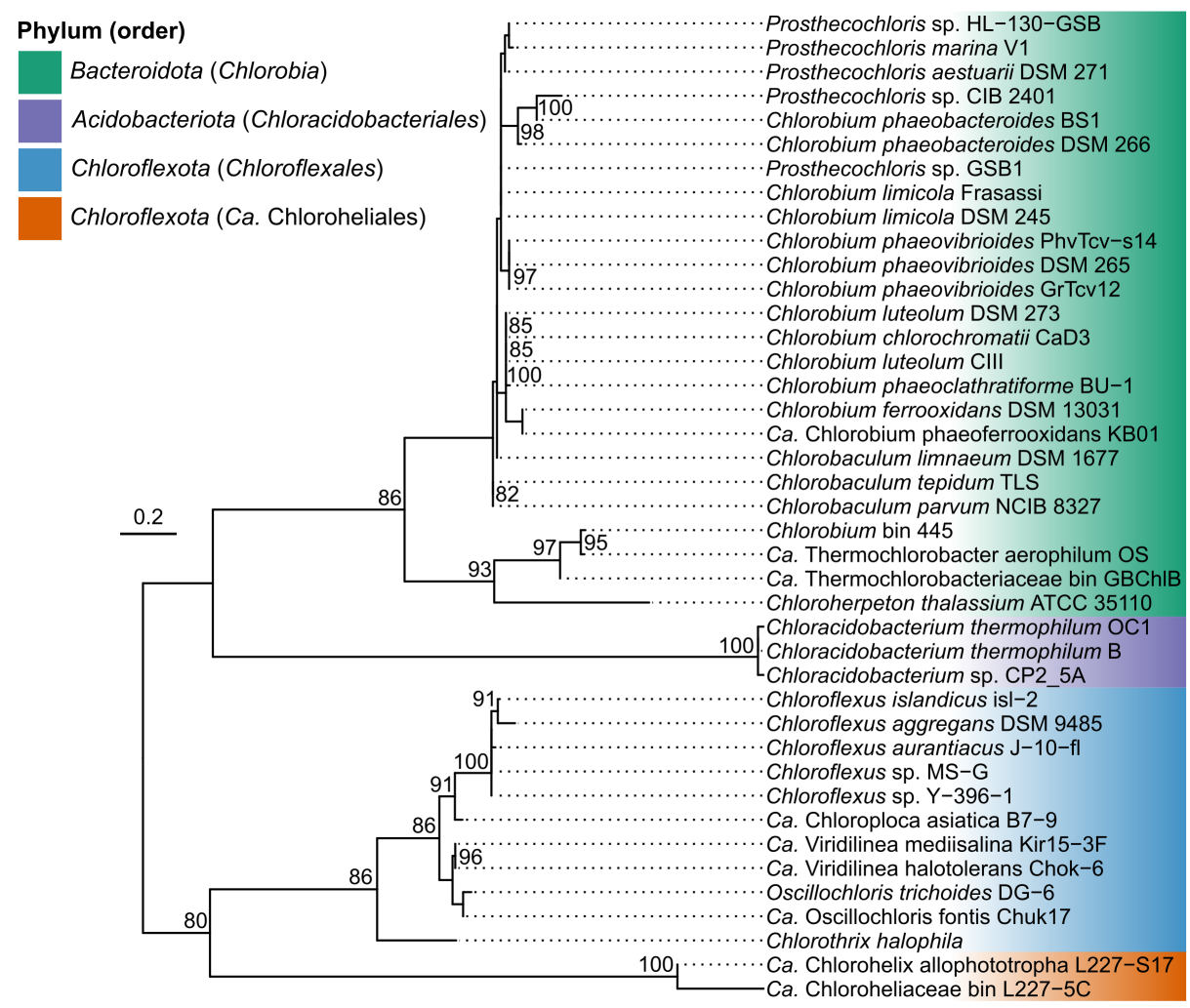

Extended Data Fig. 8 | Maximum likelihood phylogeny of CsmA predicted protein sequences. A simplified depiction of the same phylogeny is shown in Fig. 4a. The phylogeny is midpoint rooted, and ultrafast bootstrap values of at least 80/100 are shown. The scale bar represents the expected proportion of amino acid change across the 74-residue masked sequence alignment (Extended Data Table 2). 
bioRxiv preprint doi: https://doi.org/10.1101/2020.07.07.190934; this version posted August 7, 2021. The copyright holder for this preprint (which was not certified by peer review) is the author/funder, who has granted bioRxiv a license to display the preprint in perpetuity. It is made available under aCC-BY-NC-ND 4.0 International license.

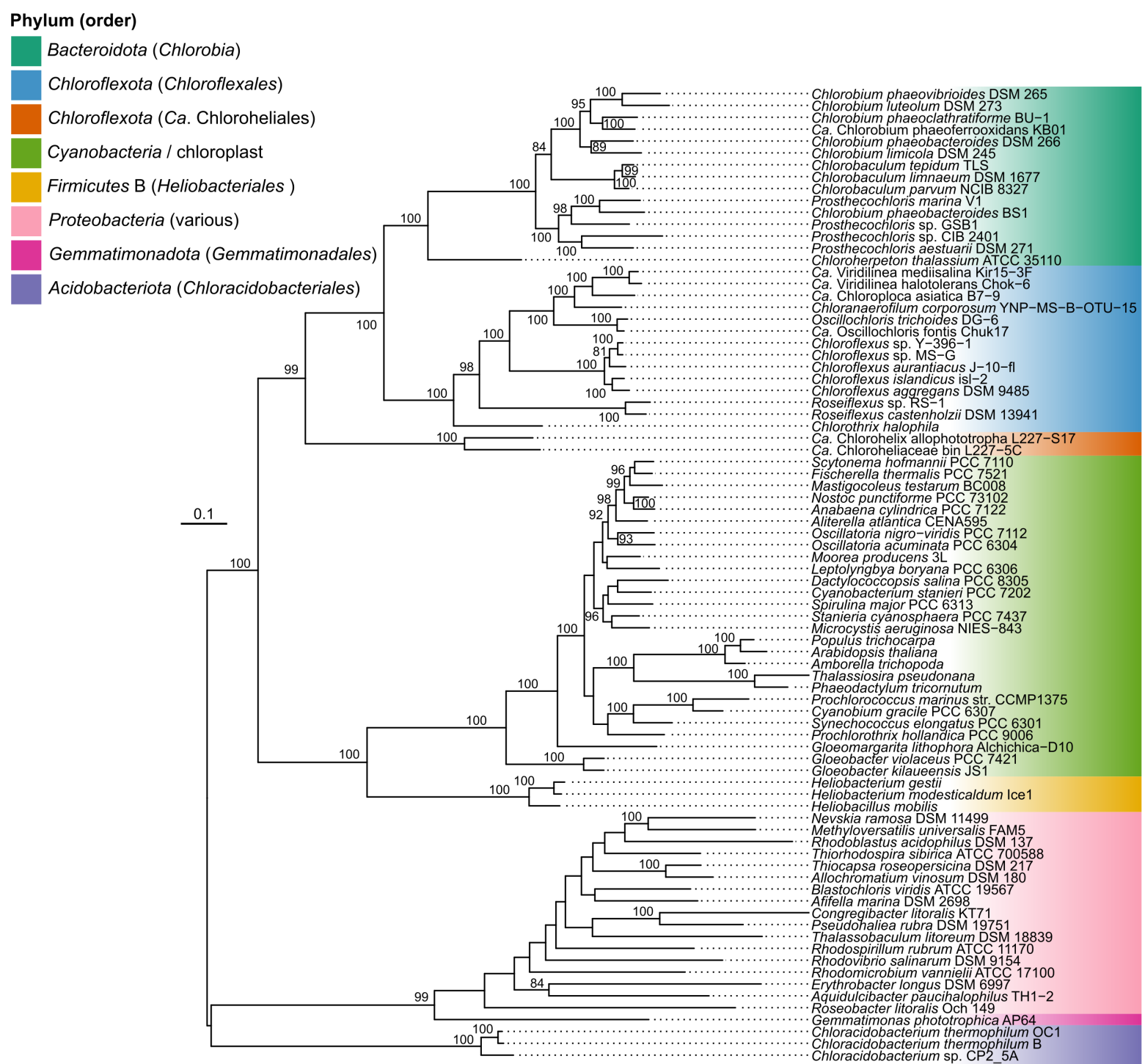

Extended Data Fig. 9 | Maximum likelihood phylogeny of BchIDH/ChIIDH protein sequences. A simplified depiction of the same phylogeny is shown in Fig. 4b. The phylogeny is midpoint rooted, and ultrafast bootstrap values of at least $93080 / 100$ are shown. The scale bar represents the expected proportion of amino acid change across the 1702-residue masked sequence alignment (Extended Data Table 2). No sequences associated with the Eremiobacterota phylum are shown, because the complete BchIDH gene set could not be detected in any of the genome bins available from that phylum using bidirectional BLASTP. 
bioRxiv preprint doi: https://doi.org/10.1101/2020.07.07.190934; this version posted August 7, 2021. The copyright holder for this preprint (which was not certified by peer review) is the author/funder, who has granted bioRxiv a license to display the preprint in perpetuity. It is made available under aCC-BY-NC-ND 4.0 International license.


Extended Data Fig. 10 | Maximum likelihood phylogeny of predicted protein sequences of the paralogs

BchLNB/ChILNB and BchXYZ. The phylogeny is midpoint rooted, and ultrafast bootstrap values of at least 80/100 are shown. The scale bar represents the expected proportion of amino acid change across the 819-residue masked sequence alignment (Extended Data Table 2). One sequence among the Chloroflexales cluster for BchXYZ, associated with "Ca.

940 Thermofonsia bin JP3-7", belongs to a genome bin that places in the basal clades of the Chloroflexota phylum (see Fig. 2) and is thus marked with an asterisk. 


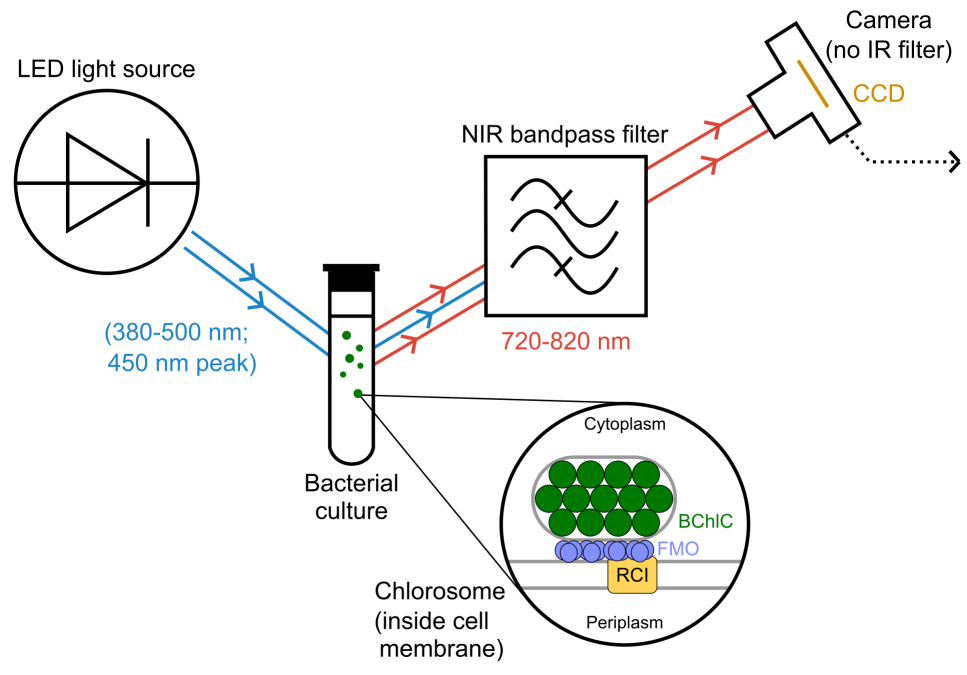

Extended Data Fig. 11 | Schematic of a pigment fluorescence detection system optimized for bacteriochlorophyll $c$.

945 Light is depicted as straight lines with arrows, and pigment-containing microbial biomass inside the tube is depicted as green dots. The fluorescently active chlorosome complex of an example Type I reaction center-containing cell is shown as an inset. An example photograph using the system is shown in Extended Data Fig. 3a. Abbreviations: LED, light-emitting diode; IR, infrared; NIR near infrared; $\mathrm{CCD}$, charge-coupled device; $\mathrm{BChlC}$, bacteriochlorophyll $c$; FMO, Fenna-MatthewsOlson protein; RCI, Type I photosynthetic reaction center. 
bioRxiv preprint doi: https://doi.org/10.1101/2020.07.07.190934; this version posted August 7, 2021. The copyright holder for this preprint (which was not certified by peer review) is the author/funder, who has granted bioRxiv a license to display the preprint in perpetuity. It is made available under aCC-BY-NC-ND 4.0 International license.

Extended Data Table 1 | Genes potentially involved in phototrophy or carbon/nitrogen fixation among genomes of "Ca. Chloroheliales" members recovered in this study. The GenBank accession numbers for the predicted primary sequence of each gene are shown. Results correspond to those shown in Fig. 2 except that homologs associated with the incomplete 3-hydroxypropionate bicycle are omitted for clarity. Raw results of bidirectional BLASTP are available in the code repository associated with this work, and queries for bidirectional BLASTP are summarized in Supplementary Data 2.

\begin{tabular}{|c|c|c|c|}
\hline Protein set & Gene & $\begin{array}{l}\text { "Ca. Chx } \\
\text { allophototropha" }\end{array}$ & $\begin{array}{l}\text { "Ca. Chloroheliaceae } \\
\text { bin L227-5C" }\end{array}$ \\
\hline \multirow{2}{*}{ Type I reaction center-associated } & $f m o A$ & NWJ48165.1 & NWJ94304.1 \\
\hline & $p s c A$ & NWJ45109.1 & NWJ94303.1 \\
\hline \multirow{3}{*}{ Chlorosome structure and assembly } & $\operatorname{csm} A$ & NWJ45202.1 & NWJ94716.1 \\
\hline & $\operatorname{csmM}$ & NWJ45728.1 & N/A \\
\hline & $\operatorname{csm} Y$ & NWJ47824.1 & NWJ94536.1 \\
\hline \multirow{19}{*}{ (Bacterio)chlorophyll synthesis } & bchl & NWJ45782.1 & NWJ94300.1 \\
\hline & $b c h D$ & NWJ47387.1 & NWJ94298.1 \\
\hline & $b c h H$ & NWJ47295.1 & NWJ97069.1 \\
\hline & bchM & NWJ47294.1 & NWJ95706.1 \\
\hline & bchJ & NWJ45810.1 & NWJ97609.1 \\
\hline & bchE & NWJ45809.1 & NWJ97608.1 \\
\hline & $\operatorname{acs} F$ & NWJ48920.1 & N/A \\
\hline & $b c h N$ & NWJ46338.1 & NWJ94750.1 \\
\hline & $b c h B$ & NWJ46337.1 & NWJ94751.1 \\
\hline & $b c h L$ & NWJ46336.1 & NWJ94752.1 \\
\hline & $b c h F$ & NWJ46647.1 & NWJ97746.1 \\
\hline & $b c h X$ & NWJ47811.1 & NWJ94280.1 \\
\hline & $b c h Y$ & NWJ47812.1 & NWJ94279.1 \\
\hline & $b c h Z$ & NWJ47813.1 & NWJ94278.1 \\
\hline & $b c h C$ & NWJ44534.1 & NWJ98808.1 \\
\hline & $b c h G$ & NWJ46528.1 & NWJ94994.1 \\
\hline & $b c h P$ & NWJ45618.1 & NWJ97704.1 \\
\hline & $b c h U$ & NWJ44614.1 & NWJ96331.1 \\
\hline & $b c h K$ & NWJ44593.1 & NWJ96281.1 \\
\hline \multirow{2}{*}{ CBB (Calvin) cycle } & $c b b P$ & NWJ44408.1 & NWJ96365.1 \\
\hline & $c b b L$ & NWJ44404.1 & NWJ96371.1 \\
\hline \multirow{2}{*}{ Nitrogen fixation } & nifH & NWJ48757.1 & NWJ96804.1 \\
\hline & nifD & NWJ48753.1 & NWJ96800.1 \\
\hline
\end{tabular}


Extended Data Table 2 | Properties of sequence alignments and phylogenetic trees presented in this study. The

960 unmasked alignment length of the core protein set was not available due to the constraints of the software pipeline used to generate the final masked alignment.

\begin{tabular}{llll}
\hline Protein set & $\begin{array}{l}\text { Alignment } \\
\text { length } \\
\text { (unmasked) }\end{array}$ & $\begin{array}{l}\text { Alignment } \\
\text { length } \\
\text { (masked) }\end{array}$ & $\begin{array}{l}\text { Evolutionary } \\
\text { rate model used }\end{array}$ \\
\hline Core proteins (Ch/oroflexota phylum) & $\mathrm{N} / \mathrm{A}$ & 11613 & $\mathrm{LG+F+R6}$ \\
Type I reaction center (PsaA/PsaB/PscA/PshA) & 1292 & 548 & $\mathrm{LG+F+G4}$ \\
FmoA & 399 & 356 & $\mathrm{LG}+\mathrm{G} 4$ \\
$\mathrm{CbbL}$ & 644 & 412 & $\mathrm{LG}+\mathrm{I}+\mathrm{G} 4$ \\
$\mathrm{CsmA}$ & 89 & 74 & $\mathrm{LG}+\mathrm{F}+\mathrm{G} 4$ \\
$\mathrm{BchlDH} / \mathrm{Ch}$ IIDH & 3156 & 1702 & $\mathrm{LG}+\mathrm{F}+\mathrm{+}+\mathrm{G} 4$ \\
BchLNB/ChILNB/BchXYZ & 2010 & 819 & $\mathrm{LG}+\mathrm{F}+\mathrm{I}+\mathrm{G} 4$ \\
\hline
\end{tabular}

